\title{
A 1D Analysis of Direct and Indirect Drive Target Performance for Planar Hydrodynamics Experiments on the NIF
}

\author{
M.J. Edwards
}

August 3, 2000

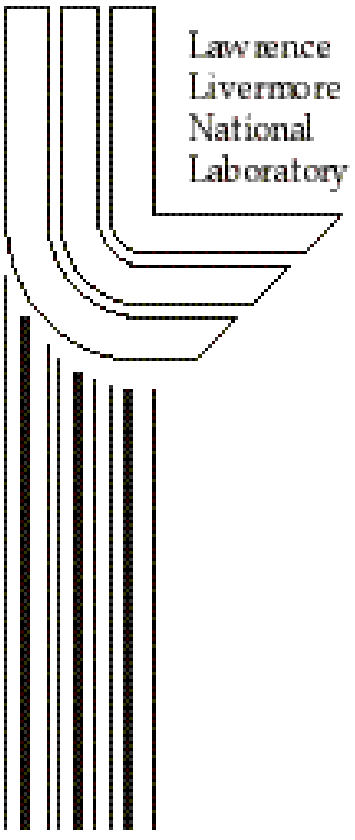




\section{DISCLAIMER}

This document was prepared as an account of work sponsored by an agency of the United States Government. Neither the United States Government nor the University of California nor any of their employees, makes any warranty, express or implied, or assumes any legal liability or responsibility for the accuracy, completeness, or usefulness of any information, apparatus, product, or process disclosed, or represents that its use would not infringe privately owned rights. Reference herein to any specific commercial product, process, or service by trade name, trademark, manufacturer, or otherwise, does not necessarily constitute or imply its endorsement, recommendation, or favoring by the United States Government or the University of California. The views and opinions of authors expressed herein do not necessarily state or reflect those of the United States Government or the University of California, and shall not be used for advertising or product endorsement purposes.

Work performed under the auspices of the U. S. Department of Energy by the University of California Lawrence Livermore National Laboratory under Contract W-7405-Eng-48.

This report has been reproduced

directly from the best available copy.

Available to DOE and DOE contractors from the

Office of Scientific and Technical Information

P.O. Box 62, Oak Ridge, TN 37831

Prices available from (423) 576-8401

http:/ /apollo.osti.gov/bridge/

Available to the public from the National Technical Information Service

U.S. Department of Commerce 5285 Port Royal Rd., Springfield, VA 22161

http://www.ntis.gov/

OR

Lawrence Livermore National Laboratory

Technical Information Department's Digital Library

http://www.llnl.gov/tid/Library.html 


\title{
A 1D Analysis of Direct and Indirect Drive Target Performance for Planar Hydrodynamics Experiments on the NIF
}

\author{
M. John Edwards \\ Lawrence Livermore National Laboratory
}

\begin{abstract}
The 1D performance of laser or X-ray driven targets to study phenomena such as the the Richtmyer-Meshkov instability in a single, steady shock, step down in density system has been described by a simple model based on 1D hydrodynamics. It is shown that the distance the interface travels under constant velocity conditions is a multiple of the separation between the ablation and shock front, and that this multiple depends on the density ratio at the interface, and the equations of states of the two materials. The model is applied to NIF with the aid of 1D hydrocode simulations to predict the ablation-shock separation. It is found that if adequate interface planarity can be maintained over an experimental length equal to the focal spot diameter, direct drive may out-perform indirect drive by up to $\sim$ factor 2 at the same pulse length and typically $\geq 2$ at the same ablation pressure. This depends on the ability to control 2D effects in the directly driven targets (critically), and on the optimum hohlraum performance achievable for these experiments, rather than the achievable performance used for the study. It is predicted that several $\mathrm{mm}$ of constant velocity interface travel are potentially achievable on NIF, and that this is only weakly dependent on the available energy. The 1D model and its application are described. Uncertainties surrounding the predictions are discussed, and means to resolve them outlined.
\end{abstract}




\subsection{Introduction}

Using high power lasers as drivers for fundamental physics studies is now a well established and mature science. In recent times there have been many instances when high quality, quantitative data obtained using lasers have made a significant impact on fundamental physics understanding. In most cases, these data were obtained using the indirect drive method in which the laser light is first used to heat a hohlraum providing a source of quasi-thermal X-rays for driving the experiment. Although this is intrinsically less efficient than coupling directly to a target (direct drive), there are at least two very important reasons why this was done. Either the experiment required an X-ray environment, or because the hohlraum effectively smooths the relatively large, high frequency spatial non-uniformities in the laser beam providing an effectively spatially smooth X-ray drive source. In most cases this has been more than adequate to perform well controlled experiments. This approach has been extremely successful. However, with advances in theoretical and practical control over laser beam propagation and uniformity, driven principally by laser fusion requirements, there is now reason to reexamine the direct drive approach. This is particularly timely as campaigns are running down on Nova, and transferring to other facilities, in particular Omega, and preparations are beginning for experiments on NIF.

The type of experiments for which direct drive might be of value are those in which the pressure generation region is decoupled from the experiment itself, and nature of the driver is of little consequence. These are principally shock driven type experiments in areas such as material properties (e.g equation of state (EOS), strength, spall), and hydrodynamics, in particular concerning instabilities and mix. Each has specific design criteria, and objectives, and must be considered separately, but in general bigger is better, and for a given driver, size is in effect traded against pressure. For this study we have selected the Richmyer-Meshkov instability for which to make a direct indirect drive performance comparison. There are several reasons for this. First, it remains controversial and as such an active area of research. Better (non) linear data are needed as well as information on the transition to turbulence. Second, targets and their behavior exhibit close similarities to other buried interface experiments such as those to measure EOS so that any analysis isof more general value. Finally, there is a need to develop an understanding of basic target dynamics to help target design, as well as to make a useful comparison between direct and indirect drive. This should help guide preparatory computational studies and experimental work on Omega, and assist planning for NIF.

In this paper, we develop a framework for defining optimized 1D target designs for studying Richtmyer-Meshkov instability in single shock, single interface systems, although the concepts and methods are readily extendable to more complex systems. We quantify these for the NIF for both direct and indirect drive, allowing a comparison of the relative performance of the two methods to be made at the same time as pointing out the main uncertainties that need to be addressed and resolved. This provides a good starting point for more detailed and refined design simulations, and a guide for design of more complex systems. 
The paper is arranged as follows. In section 2 we propose an optimized scenario and develop a simple theoretical framework to describe target dynamics. This predicts a simple parameter for quantitatively characterizing 1D performance. This is then verified with idealized hydrocode simulations. Achievable drive conditions for NIF are considered in section 3. Section 4 deals with applying the simple model to NIF by first deriving power laws for the characterization parameter as a function of drive parameters using 1D hydrocode simulations, and then combining these with the results of sections 2 and 3. This results in predictions for NIF performance in both direct and indirect mode. Prior to concluding, implications on performance derived from Mach number considerations are discussed in section 5. 


\subsection{Optimized 1D target design}

Hydrodynamic instabilities grow with time, or in the laboratory frame, with distance traveled. In the linear regime, the Richtmyer-Meshkov instability grows in proportion to the distance traveled by the interface. Linear, non-linear, and turbulent growth are all of interest, and much remains uncertain about the instability and transitions between the various regimes. Laser experiments provide the capability of accessing high Mach number, compressible flows, but suffer from small scales, limiting the amount of growth achievable under well-controlled conditions (constant interface velocity conditions). Maximizing the distance traveled under such conditions for a given laser is important for optimizing the experiment and its impact on theoretical and modeling developments. In this section we justify a near optimum configuration, and then proceed to quantify the target design in general terms.

\subsection{An optimum configuration - simple target dynamics}

We consider a simple target consisting of an ablator/pusher of $1 \mathrm{~g} / \mathrm{cc} \mathrm{CH}$, driving a low density foam, with the instability developing at the interface between them (fig 1). We suppose the laser or $\mathrm{X}$-rays ablate the outer region of the $\mathrm{CH}$ in such a way as to drive a constant velocity (pressure) shock wave towards the interface. The target behavior is depicted schematically in figure 2 . As the shock wave passes across the interface into the lower density material it speeds up to a new constant velocity, with the interface following at some fraction of this depending on the EOSs of the materials. At the same time, a rarefaction propagates back into the compressed pusher towards the ablation front. On reaching the ablation front this wave is reflected back towards the interface. It is only when this wave catches up with the interface that the interface becomes aware of a change in conditions, and undergoes changes in velocity. This ends the well-controlled, constant velocity period.

How, and when, the period of constant interface velocity ends depends on when the "piston" (laser or X-rays) turns off. It is straightforward to show that the thickness of the compressed pusher is a governing parameter in determining the duration of the constant interface velocity period for given driver pressure, and pusher material. Maximizing this is important. The rate at which the ablation front eats into the compressed pusher is slower than the local sound speed so maintaining the drive pressure until the interface rarefaction hits the ablation front achieves a maximum effective separation (see figure 3 ). Maintaining the drive after reflection off the ablation front causes the pressure there to be higher than it wants to be and results in a compression wave returning towards the interface, rather than a rarefaction, but does not delay the progress of the wave. A compression wave would eventually end the constant velocity period with an abrupt interface acceleration, whereas the reflected rarefaction would result in a gradual decay in the velocity of the interface. This is shown schematically in figure 4 . Either might be desirable depending on the experiment, and the latter may actually still be usable as "adequately" constant velocity. We maintain that continuing to drive the experiment after the interface rarefaction reflects off the ablation front is wasteful unless the 
compression wave is required, and that the optimum drive ends when reflection occurs for a simple single shock system.

Before quantifying such optimized target parameters, it is noted that there may be a drive profile that can minimize the effect of the reflected wave on the interface, and effectively do better than the configuration described above.

\subsection{Optimized $1 D$ target parameters - general determination}

The target dynamics are depicted schematically in figure 2. After the shock has passed through the interface, the target consists of: A) shocked pusher between the ablation front and head of the rarefaction; B) the rarefaction wave; C) a region of constant velocity, density and pressure following the interface; D) shocked foam, and E) unshocked foam. We aim to determine the time at which the reflected wave catches the interface, and equivalently the distance propagated by the interface during this time. The head of the wave travels first through $\mathrm{A}$, reflects off the ablation front, returns through $\mathrm{B}$ then $\mathrm{C}$, finally striking the interface. In this section the starting point, $t=0$, is taken to be the time at which the shock wave strikes the undisturbed interface. It is then straightforward to extrapolate to the initial drive and target conditions.

First, we determine the trajectory of the interface rarefaction, and reflected wave (B) since the passage through $\mathrm{A}$ is trivial. We work primarily in the frame travelling with the shocked pusher material, $\Sigma_{p}$, at speed $u_{p}$ in the laboratory frame. In this frame the interface rarefaction travels back towards the ablation front at the local sound speed, $\mathrm{c}_{\mathrm{o}}$. Upon reflection, the wave acquires velocity $\mathrm{w}=\mathrm{u}+\mathrm{c}$, where $\mathrm{u}$ and $\mathrm{c}$ are the local fluid velocity and sound speed respectively in the rarefaction wave (B). From the theory of simple waves

$$
\begin{aligned}
u & =\frac{2}{\gamma-1}\left(c_{o}-c\right)=\frac{x}{t}+c \\
\Rightarrow c & =\frac{\gamma-1}{\gamma+1}\left(\frac{2}{\gamma-1} c_{o}-\frac{x}{t}\right)
\end{aligned}
$$

The velocity, $w$, of the reflected wave is just $d x / d t=u+c=2 c+x / t$. Substituting $c$ from above and integrating we obtain for the displacement of the head of the wave

$$
x_{f}=\frac{2}{\gamma-1} c_{o} t-\frac{\gamma+1}{\gamma-1} L\left(\frac{c_{o} t}{L}\right)^{\frac{3-\gamma}{\gamma+1}}
$$

In the laboratory frame 


$$
x_{f-l a b}=\frac{2}{\gamma-1} c_{o} t-\frac{\gamma+1}{\gamma-1} L\left(\frac{c_{o} t}{L}\right)^{\frac{3-\gamma}{\gamma+1}}+u_{p} t
$$

where $\mathrm{L}$ is the effective width of the shocked pusher, to which we shall return later.

We now calculate the time at which the reflected wave catches the point where the rarefaction wave $(\mathrm{B})$ connects to the constant velocity portion $(\mathrm{C})$ behind the transmitted shock wave. In the laboratory frame, and for strong shocks, the interface travels at $u_{i}$ which can be determined by standard methods as

$$
u_{i}=\left(\frac{2}{\gamma_{f}+1} \frac{\gamma+1}{\gamma(\gamma-1)}\right)^{1 / 2}\left(\frac{q}{\eta}\right)^{1 / 2} c_{o}
$$

where

$$
\left(\frac{2}{\gamma_{f}+1}\right)^{1 / 2}\left(\frac{q}{\eta}\right)^{1 / 2}=\left(\frac{2}{\gamma_{f}+1}\right)^{1 / 2}+\left(\frac{2}{\gamma-1}\right)\left[\frac{\gamma(\gamma-1)}{\gamma+1}\right]^{1 / 2}\left(1-q^{\frac{\gamma-1}{2 \gamma}}\right)
$$

$\gamma_{\mathrm{f}}$ is the specific heat ratio for the foam, $\eta$ is the pre-shock foam to pusher density ratio, and $q$ is the ratio of the post-shock interface pressure, $\mathrm{P}_{\mathrm{i}}$, to the drive pressure, $\mathrm{P}_{\mathrm{o}}$. Solutions to eqns 4 and 5 are shown in figure 5.

The displacement of the point connecting the two regions $\mathrm{B}$ and $\mathrm{C}$ can be obtained from the theory of simple waves, as that point in the rarefaction with velocity $u_{i}-u_{p}$ in $\Sigma_{p}$. This is

$$
x_{i}=\left(\left[u_{i}-u_{p}\right]-c_{i}\right) t \quad \text { with } \quad\left[u_{i}-u_{p}\right]=\frac{2}{\gamma-1}\left(c_{o}-c_{i}\right)
$$

From the adiabatic condition we have

$$
\frac{c_{i}}{c_{o}}=\left(\frac{P_{i}}{P_{o}}\right)^{\frac{\gamma-1}{2 \gamma}}=q^{\frac{\gamma-1}{2 \gamma}}
$$

so that finally we obtain for the displacement of this point in the laboratory frame 


$$
x_{i-l a b}=x_{i}+u_{p} t=\left\{\frac{2}{\gamma-1}-\frac{\gamma+1}{\gamma-1} q^{\frac{\gamma-1}{\gamma}}+\sqrt{\frac{2}{\gamma(\gamma-1)}}\right\} c_{o} t
$$

It is now straightforward to determine the time, $t_{c}$, at which the reflected wave reaches this point by setting $\mathrm{x}_{\mathrm{f}-\mathrm{lab}}=\mathrm{x}_{\mathrm{i}-\mathrm{lab}}=\mathrm{x}_{\mathrm{i}-\mathrm{ab}-\mathrm{c}}$

$$
t_{\mathrm{c}}=\frac{L}{c_{o}}\left(\frac{c_{o} t_{c}}{L}\right)^{\frac{3-\gamma}{\gamma+1}} q^{\frac{1-\gamma}{2 \gamma}}=\frac{L}{c_{o}} q^{-\frac{\gamma+1}{4 \gamma}}
$$

At this time, the interface will have traveled a distance $\mathrm{u}_{\mathrm{i}} \mathrm{t}_{\mathrm{c}}$ from its initial position, and the reflected wave now approaches the interface at $c_{i}$ in the frame moving with the interface (at velocity $\mathrm{u}_{\mathrm{i}}$ ). The time it takes the wave to traverse this separation is just $\left(\mathrm{u}_{\mathrm{i}} \mathrm{t}_{\mathrm{c}}-\mathrm{x}_{\mathrm{i}-\text {-ab-c }}\right) / \mathrm{c}_{\mathrm{i}}$. If we note that $\mathrm{x}_{\mathrm{i}-\mathrm{ab}}=\mathrm{x}_{\mathrm{i}}+\mathrm{u}_{\mathrm{p}} \mathrm{t}$, then from eqn. $6 \mathrm{x}_{\mathrm{i}-\mathrm{ab}-\mathrm{c}}=\left(\mathrm{u}_{\mathrm{i}}-\mathrm{c}_{\mathrm{i}}\right) \mathrm{t}_{\mathrm{c}}$, and the time to traverse the constant velocity section is also clearly $t_{c}$. Therefore, the time at which the reflected wave reaches the interface is

$$
t_{\text {coll }}=2 t_{c}=2 \frac{L}{c_{o}} q^{-\frac{\gamma+1}{\psi_{l}}}
$$

Finally the displacement of the interface relative to its initial position at the time when the reflected wave strikes the interface is

$$
L_{\mathrm{nt}}=u_{i} t_{\text {coll }}=\left(\frac{2}{\gamma_{f}+1} \frac{\gamma+1}{\gamma(\gamma-1)}\right)^{1 / 2}\left(\frac{q}{\eta}\right)^{1 / 2} q^{-\frac{\gamma+1}{4 \gamma}} 2 L
$$

It only remains to specify L. In the picture of the optimized target, $\mathrm{L}$ is the width of the compressed pusher seen by the rarefaction before it reflects off the ablation front. Thus if the ablation velocity in $\Sigma_{\mathrm{p}}$ is $\mathrm{u}_{\mathrm{a}}$, the initial pusher width $\mathrm{L}_{\mathrm{o}}$, the shock speed $\mathrm{v}_{\mathrm{s}}$ in the laboratory frame, and the mass ablation rate $\dot{m}$, the width of the pusher when the shock strikes the interface is 


$$
L_{s}=\frac{\gamma-1}{\gamma+1} L_{o}-u_{a} \frac{L_{o}}{v_{s}}
$$

and the width seen by the rarefaction is

$$
L=\frac{L_{s}}{u_{a}+c_{o}} c_{o}=\frac{\gamma-1}{\gamma+1} \frac{1-\dot{m} / \rho_{o} v_{s}}{1+\frac{u_{a} / c_{o}}{\gamma-1} \rho_{o}}
$$

The drive pulse length is

$$
t_{D}=t_{s}+\frac{L_{s}}{u_{a}+c_{o}}=\frac{L_{o}}{v_{s}}\left\{1+\sqrt{\frac{\gamma-1}{2 \gamma}} \frac{\left[1-\dot{m} / \rho_{o} v_{s}\right]}{\left[1+u_{a} / c_{o}\right]}\right\}
$$

where we have used the relationship $v_{s}=\left[\frac{(\gamma+1)^{2}}{2 \gamma(\gamma-1)}\right]^{1 / 2} c_{o}$

Below we collect these results together for $\gamma=\gamma_{\mathrm{t}}=5 / 3$, and later use them in estimating the performance of direct and indirect drive configurations.

$$
\begin{aligned}
x_{f} & =3 c_{o} t-4 L\left(\frac{c_{o} t}{L}\right)^{1 / 2} \\
x_{f-l a b} & =3 c_{o} t-4 L\left(\frac{c_{o} t}{L}\right)^{1 / 2}+u_{p} t \\
u_{i} & =3 / \sqrt{5}\left(\frac{q}{\eta}\right)^{1 / 2} c_{o} \\
\left(\frac{q}{\eta}\right)^{1 / 2} & =1+\sqrt{5}\left[1-q^{1 / 5}\right] \\
t_{\mathrm{c}} & =\frac{L}{c_{o}} q^{-2 / 5} \\
L_{\mathrm{nt}} & =3 / \sqrt{5}\left(\frac{q}{\eta}\right)^{1 / 2} q^{-2 / 5} 2 L
\end{aligned}
$$




\subsection{Model verification \& implications}

The model presented above indicates that the system is entirely characterized by, $L, c_{0}, \eta$, and $\gamma$. In addition, it predicts that the interface travel under constant velocity conditions is directly proportional to L. A series of non-ablative, model 1D Hyades simulations has been conducted to verify these results. The "target" consists of a $1 \mathrm{~g} / \mathrm{cc}$ pusher of initial width $x_{0}$, and a foam of density $0.1 \mathrm{~g} / \mathrm{cc}(\eta=0.1 \Rightarrow \mathrm{q} \approx 0.24)$. Both pusher and foam were assumed to behave as perfect gases with $\gamma=5 / 3$, so that $L=x_{0} / 4$. A schematic is shown in figure 6.

A constant boundary pressure, $\mathrm{P}_{\mathrm{D}}$, was applied to the free surface of the pusher, and removed when the rarefaction, reflected from the interface, arrived at the piston, thus emulating the optimized situation described above. The shock velocity is given by $\mathrm{v}_{\mathrm{s}}=$ $\left[(\gamma+1) / 2 . \mathrm{P}_{\mathrm{D}} / \rho_{\mathrm{o}}\right]^{1 / 2}$, and the time for the shock to traverse the pusher is $\mathrm{t}_{\mathrm{s}}=\mathrm{x}_{\mathrm{o}} / \mathrm{v}_{\mathrm{s}}$. The sound speed, $c_{o}=\left(\gamma \mathrm{P}_{\mathrm{D}} / 4 \rho_{\mathrm{o}}\right)^{1 / 2}$ was varied by changing $\mathrm{P}_{\mathrm{D}}$, while $\mathrm{L}$ was varied by altering $\mathrm{x}_{\mathrm{o}}$. The simulations were followed beyond the time when the reflected rarefaction struck the interface. The nominal case selected was $P_{D}=240$ Mbar, $x_{o}=400 \mu \mathrm{m}(L=100 \mu \mathrm{m})$, so that $\mathrm{c}_{\mathrm{o}}=100 \mu \mathrm{m} / \mathrm{ns}, \mathrm{v}_{\mathrm{s}}=178 \mu \mathrm{m} / \mathrm{ns}$. The model predictions are tabulated below for a variety of configurations.

\begin{tabular}{|l|l|l|l|l|l|l|l|l|}
\hline & $\begin{array}{c}\mathrm{x}_{\mathrm{o}} \\
(\mu \mathrm{m})\end{array}$ & $\begin{array}{c}\mathrm{L} \\
(\mu \mathrm{m})\end{array}$ & $\begin{array}{c}\mathrm{P}_{\mathrm{D}} \\
(\mathrm{Mbar})\end{array}$ & $\begin{array}{c}\mathrm{c}_{\mathrm{o}} \\
(\mu \mathrm{m} / \mathrm{ns})\end{array}$ & $\mathrm{t}_{\mathrm{s}}(\mathrm{ns})$ & $\begin{array}{c}\mathrm{t}_{\text {coll }} \\
(\mathrm{ns})\end{array}$ & $\begin{array}{c}\mathrm{u}_{\mathrm{i}} \\
(\mu \mathrm{m} / \mathrm{ns})\end{array}$ & $\begin{array}{c}\Delta \mathrm{x}_{\mathrm{i}}=\mathrm{L}_{\text {int }} \\
(\mu \mathrm{m})\end{array}$ \\
\hline $\mathrm{A}$ & 400 & 100 & 240 & 100 & 2.24 & 3.54 & 207 & 733 \\
\hline $\mathrm{B}$ & 400 & 100 & 960 & 200 & 1.12 & 1.77 & 415 & 733 \\
\hline $\mathrm{C}$ & 400 & 100 & 60 & 50 & 4.48 & 7.08 & 103 & 733 \\
\hline $\mathrm{D}$ & 200 & 50 & 240 & 100 & 1.12 & 1.77 & 207 & 366 \\
\hline $\mathrm{E}$ & 800 & 200 & 240 & 100 & 4.48 & 7.08 & 207 & 1465 \\
\hline
\end{tabular}

The results of the simulations are shown graphically in figure 7. As can be seen, the simulations reproduce the predictions almost exactly in all cases. Furthermore, the scaling predicted by the model is born out in the simulations. For example, A-C should all result in the same $\mathrm{L}_{\text {int }}$ although the time scale over which this happens is quite different. Also, a factor 2 change in $L(D \& E)$ is faithfully reproduced in $\mathrm{L}_{\text {int }}$ as expected. This implies that if $\mathrm{L}_{\text {int }}$ were the only consideration, the time scale of the experiment is irrelevant provided $\mathrm{L}$ is preserved.

Finally, it is noted that the expression for $\mathrm{L}_{\text {int }}$ is only weakly dependent on $\mathrm{q}$ and varies $\mathrm{q}^{1 / 10} / \eta^{1 / 2}$. Reducing the density ratio to 0.01 increases $\mathrm{L}_{\text {int }}$ by $\sim 2.65$. Further, as $\eta->0$ $(\mathrm{q} / \eta)^{1 / 2}->1+\sqrt{ } 5$ and the model predicts $\mathrm{u}_{\mathrm{i}}>3(1+1 / \sqrt{5}) \mathrm{c}_{\mathrm{o}}=3 \mathrm{c}_{\mathrm{o}}+\mathrm{u}_{\mathrm{p}}$ which is exactly the solution expected. 


\subsection{NIF Driver Performance}

\subsection{Available energy}

For the planar experiments considered here it is assumed that all 96 beams from one end of NIF can be used in an effective direct drive experiment. While up to the entire 192 beams might be used for indirect geometry, the preferred configuration is also taken to be single ended into a half hohlraum, providing roughly equivalent performance and greatly enhanced backlighting capability (fig 8).

In practice, and for the achievable drive conditions, 1D target performance improves with the intensity and duration of the drive radiation. It is expected that most experiments of this type will use $\geq 5 \mathrm{~ns}$ laser pulses. The maximum laser energy deliverable to the target by 192 beams in $3 \omega$ is taken to be $2.5 \mathrm{MJ}$. This is consistent with recent predictions of laser performance at $5 \mathrm{~ns}$ and $13 \mathrm{~ns}$. It is assumed that beam staggering can be used to achieve laser pulse durations above $13 \mathrm{~ns}$. Further, it is assumed that phase plates can be fabricated to produce a reasonably flat spatial profile containing most of the energy in a single spot.

\subsection{Direct drive}

The above assumptions place almost no constraints on the achievable laser intensities of interest for directly driving planar hydrodynamics experiments. The maximum intensity achievable is

$$
I_{\max }=160\left(\frac{E_{M J}}{1.25}\right) \frac{1}{t_{n s} \phi_{m m}^{2}} \quad 10^{15} \mathrm{Wcm}^{-2}
$$

where $\phi$ is the focal spot diameter. The limiting factors will be $2 \mathrm{D}$ effects in the hydrodynamics of the package causing distortion of interface planarity, and 2D effects in laser target coupling which could both distort the interface, and result in less efficient pressure generation. While detailed 2D simulations should go a long way towards quantifying these effects, it will not be possible to determine the influence of laser plasma instabilities for 10s ns pulses until experiments commence. For now we assume there will be no significant deleterious limitations.

\subsection{Indirect drive}

In the case of indirect drive past experience with laser plasma interactions imply a limit to the hohlraum filling density allowable before the laser plasma interaction undergoes catastrophic instabilities. This leads to a relationship between the minimum scale of the hohlraum permissible as a function of laser energy and pulse length, which in turn results 
in an upper limit to the achievable radiation temperature. It can be shown that hohlraums that fill to the same density lie approximately on a surface defined by ${ }^{1}$

$$
\frac{P t^{2}}{A^{2}} \sim \text { const }
$$

where the constant defines the value of the density, and $\mathrm{A}$ is the hohlraum wall area.

The hohlraum temperature can be approximated by balancing the laser energy converted to X-rays against energy absorbed by the hohlraum walls leading to

$$
T \sim\left(\frac{\varepsilon_{X} P t^{0.43}}{A}\right)^{0.3}
$$

where $\varepsilon_{\mathrm{X}}$ is the instantaneous X-ray conversion efficiency.

Combining the two relationships, and assuming $\varepsilon_{\mathrm{X}}$ is approximately constant, we find

$$
\begin{aligned}
& D \sim(E t)^{0.25} \\
& T \sim E^{0.15} t^{-0.321}
\end{aligned}
$$

This is important because it implies that if plasma filling governs the hohlraum performance, the experimental length scales slowly with laser energy for a given pulse length, roughly ${ }^{2} \sim \mathrm{E}^{0.25}$.

To obtain the constants in the above relationships we take filling to $1 / 4$ critical density for blue light to be appropriate

$$
\frac{P_{T W} t_{n s}^{2}}{A_{m m}^{2}} \sim 0.136
$$

where the hohlraum area is just $\pi\left(D^{2} / 2+D L\right)=\pi D^{2}(1 / 2+\eta)$ where $\mathrm{D}$ is the diameter, $\mathrm{L}$ the length and $\eta=\mathrm{L} / \mathrm{D}$. As a geometry baseline we take the hohlraum for Haan's pt NIF design: $\mathrm{D}=5.5 \mathrm{~mm}, \eta=0.86$, and a $50 \% \mathrm{LEH}$. By preserving the geometry (aspect ratios) as the hohlraum scales in size the laser beams should continue to enter the hohlraum safely. A better estimate of the hohlraum temperature, T, can be obtained by balancing the laser energy converted to X-rays against losses into the walls, the experimental package, and out through the laser entrance hole

$$
10 \varepsilon_{X} P_{L} d t=0.186 T^{3.38} t^{-0.43} A_{W} d t+1.03 T^{4}\left[A_{L E H}+A_{P H}(1-\alpha)\right] d t
$$

\footnotetext{
${ }^{1}$ This is a reasonable approximation provided the hot laser heated plasma governs the filling which is usually the case since it's velocity is much higher than that of the colder X-ray ablated plasma

${ }^{2}$ Ablation pressure scales roughly as $\mathrm{T}^{3.5}$ for low $\mathrm{Z}$ ablators, and velocity scales as the square root of this.

Thus hohlraum size and experimental length scale in a similar fashion with laser energy.
} 
where temperatures are in heV, times in $\mathrm{ns}$, areas in $\mathrm{mm}^{2}$, laser power in $\mathrm{TW}$, and energies in $\mathrm{hJ} . \varepsilon_{\mathrm{X}}$ is the instantaneous X-ray conversion efficiency, and $\alpha$ is the albedo of the experimental package (ie the fraction of energy reflected back into the hohlraum). For short $\sim 1 \mathrm{~ns}$ pulses this is typically no more than 0.1 , while for longer $\geq 10 \mathrm{~ns}$ pulses, this might reach $\sim 0.5$. The areas are given by

$$
\begin{aligned}
& A_{W}=\pi D^{2}\left\lceil\eta+\frac{1}{2}-\frac{\left(\beta^{2}+\gamma^{2}\right)}{4}\right\rfloor \\
& A_{L E H}=\frac{\pi D^{2}}{4} \beta^{2} \\
& A_{L E H}=\frac{\pi D^{2}}{4} \gamma^{2}
\end{aligned}
$$

where $\eta, \beta$ and $\gamma$, are the ratios of hohlraum length, LEH diameter, and package diameter to hohlraum diameter respectively.

Combining the above relationships for a constant temperature hohlraum, and setting $\varepsilon_{\mathrm{X}}$ $=0.7, \eta=0.86, \alpha=\beta=\gamma=0.5$ we finally obtain the following scaling laws

$$
\begin{aligned}
T & =4.44\left(\frac{\mathrm{E}_{\mathrm{MJ}}}{1.25}\right)^{0.15} t^{-0.321} \mathrm{heV} \\
D & =4.72\left(\frac{\mathrm{E}_{\mathrm{MJ}}}{1.25}\right)^{0.25} t^{0.25} \mathrm{~mm}
\end{aligned}
$$

For full energy from a single ended driver, the expression in brackets is of course unity, and the temperature expression is a very good approximation to the full energy loss equation at least up to $t \sim 100$ ns. Note that if filling limits the hohlraum performance then the drive scales very slowly with the available energy.

The above temperature equation is a good starting point, and its predictions are shown in fig 9. To test it we compute the peak temperature for several hohlraums simulated by Lasnex for a $375 \mathrm{~kJ}, 3 \mathrm{~ns}$ square laser pulse. The results are shown in fig 10. The peak temperature is rather well represented by the scaling law, albeit fortuitously. Nevertheless, it lends some credence to the simple scaling law. On further examination of the Lasnex simulations we see that the failure mechanism in the under-sized cans is roll over due to plasma build up near the LEH causing laser deposition there and X-rays to escape rather than couple inside the hohlraum. This is a different failure mechanism from filling assumed above, and results in an effective pulse length less than would be achieved in a larger hohlraum as is indeed seen in the simulations. The scaling law does not represent this situation. Furthermore, evidence on Nova suggests that filling is not the limiting performance factor for hohlraums heated by blue light, indicating that rollover might be the eventual safety valve. Therefore, while the scaling law might well reasonably predict an achievable performance, it is not obvious that it is the optimum 
performance. To better quantify the achievable drive conditions NIF experiments and detailed simulations will be required.

Finally, we note that in practice, some X-ray pulse shaping may be necessary to achieve an adequately constant interface velocity. This could be achieved with constant ablation pressure, which would necessitate a laser power falling with time after the desired temperature had been reached, which would tend to reduce slightly the temperature values predicted above. 


\subsection{Optimized NIF experimental parameters}

In this section we take the results from sections 2 and 3 and determine target dimensions and interface travel as a function of driver conditions. This allows us to compare direct drive and indirect drive performance within the assumptions and caveats discussed above. The approach we take is to fit appropriate quantities (such as ablation pressure, mass ablation rate, shock distance, and ablation front to shock front separation) predicted by 1D hyades simulations to power laws in time and laser intensity or radiation temperature. Together with the results of section 2, this provides all the information necessary to estimate the important experimental parameters, (interface travel under constant velocity conditions, $\mathrm{L}_{\mathrm{int}}$, the pusher thickness, $\mathrm{L}_{\mathrm{p}}$, and the total length of the experiment, $\mathrm{L}_{\mathrm{exp}}=$ $\mathrm{L}_{\text {int }}+\mathrm{L}_{\mathrm{p}}$ ), as functions of pulse length, and intensity or drive temperature. Section 3 then allows us to overlay achievable NIF driver performance on these results revealing optimized experimental parameters for NIF. We do this first for full NIF energy, and then consider the effect of reduced energy variants by comparing these results with those for $1 / 2$ and $1 / 4$ the total number of beams.

\subsection{Power laws}

Power law fits to hyades simulations are compared with other models and tabulated below based on the following functional form

$$
\begin{array}{lc}
\Phi_{\text {las }}=a I_{\text {las }}^{b} t^{c} & \text { laser } \\
\Phi_{R}=a T_{R}^{b} t^{c}=\alpha I_{R}^{\beta} t^{c} & \text { Xray }
\end{array}
$$

where $\Phi$ is a physical quantity such as ablation pressure, $\mathrm{I}$ is in $10^{15} \mathrm{Wcm}^{-2}, \mathrm{~T}_{\mathrm{R}}$ is in heV, and $\mathrm{t}$ is in $\mathrm{ns}$

\begin{tabular}{|l|l|l|l|}
\hline \multicolumn{5}{|c|}{ Hyades Direct Drive (NIF, $\mathbf{t} \geq \mathbf{5 n s})$} \\
\hline \multicolumn{1}{|c|}{$\Phi_{\text {las }}$} & \multicolumn{1}{c|}{$\mathrm{a}$} & \multicolumn{1}{c|}{$\mathrm{b}$} & \multicolumn{1}{c|}{$\mathrm{c}$} \\
\hline $\mathrm{P}_{\mathrm{abl}}(\mathrm{Mbar})$ & 100 & 0.79 & -0.12 \\
\hline $\mathrm{X}_{\mathrm{s}}(\mu \mathrm{m})$ & 115 & 0.377 & 0.95 \\
\hline $\mathrm{X}_{\mathrm{a}}(\mu \mathrm{m})$ & 90 & 0.42 & 0.95 \\
\hline $\mathbf{X}_{\mathrm{sa}}(\boldsymbol{\mu m})$ & $\mathbf{2 3}$ & $\mathbf{0 . 2 8}$ & $\mathbf{1 . 0}$ \\
\hline $\mathrm{m}_{\mathrm{a}}\left(\mathrm{mg} / \mathrm{cm}^{2}\right)$ & 2.65 & 0.6 & 0.65 \\
\hline
\end{tabular}

\begin{tabular}{|l|l|l|l|}
\hline \multicolumn{5}{|c|}{ Lindl Direct Drive } \\
\hline \multicolumn{1}{|c|}{$\Phi_{\text {las }}$} & \multicolumn{1}{c|}{$\mathrm{a}$} & \multicolumn{1}{c|}{$\mathrm{b}$} & \multicolumn{1}{c|}{$\mathrm{c}$} \\
\hline $\mathrm{P}_{\mathrm{abl}}(\mathrm{Mbar})$ & 80 & 0.67 & 0 \\
\hline $\mathrm{X}_{\mathrm{s}}(\mu \mathrm{m})^{\dagger}$ & 103 & 0.33 & 1.0 \\
\hline $\mathrm{X}_{\mathrm{a}}(\mu \mathrm{m})^{\dagger}$ & 80 & 0.33 & 1.0 \\
\hline $\mathbf{X}_{\mathrm{sa}}(\boldsymbol{\mu m})^{\dagger, 2}$ & $\mathbf{2 3}$ & $\mathbf{0 . 3 3}$ & $\mathbf{1 . 0}$ \\
\hline $\mathrm{m}_{\mathrm{a}}\left(\mathrm{mg} / \mathrm{cm}^{2}\right)$ & 1.05 & 0.33 & 1.0 \\
\hline
\end{tabular}




\begin{tabular}{|l|l|l|l|l|l|}
\hline \multicolumn{7}{|c|}{ Hyades X-ray Drive } \\
\hline \multicolumn{1}{|c|}{$\Phi_{\mathrm{R}}$} & $\mathrm{a}$ & $\mathrm{b}$ & $\mathrm{c}$ & \multicolumn{1}{c|}{$\alpha$} & \multicolumn{1}{c|}{$\beta$} \\
\hline $\mathrm{P}_{\mathrm{abl}}(\mathrm{Mbar})$ & 6 & 3.6 & -0.24 & 370 & 0.9 \\
\hline $\mathrm{X}_{\mathrm{s}}(\mu \mathrm{m})$ & 32 & 1.8 & 0.88 & 251 & 0.45 \\
\hline $\mathrm{X}_{\mathrm{a}}(\mu \mathrm{m})$ & 24 & 2.0 & 0.85 & 237 & 0.5 \\
\hline $\mathrm{X}_{\mathrm{sa}}(\boldsymbol{\mu m})$ & $\mathbf{7 . 7}$ & $\mathbf{1 . 0}$ & $\mathbf{1 . 0 2}$ & $\mathbf{2 4}$ & $\mathbf{0 . 2 5}$ \\
\hline $\mathrm{m}_{\mathrm{a}}\left(\mathrm{mg} / \mathrm{cm}^{2}\right)$ & 0.65 & 3.2 & 0.65 & 25.3 & 0.8 \\
\hline
\end{tabular}

\begin{tabular}{|l|l|l|l|l|l|}
\hline \multicolumn{7}{|c|}{ Lindl X-ray Drive } \\
\hline \multicolumn{1}{|c|}{$\Phi_{\mathrm{R}}$} & $\mathrm{a}$ & \multicolumn{1}{c|}{$\mathrm{b}$} & $\mathrm{c}$ & \multicolumn{1}{c|}{$\alpha$} & \multicolumn{1}{c|}{$\boldsymbol{\beta}$} \\
\hline $\mathrm{P}_{\mathrm{abl}}(\mathrm{Mbar})$ & 3 & 3.5 & 0 & 170 & 0.875 \\
\hline $\mathrm{X}_{\mathrm{s}}(\mu \mathrm{m})$ & 20 & 1.75 & 1.0 & 147 & 0.44 \\
\hline $\mathrm{X}_{\mathrm{a}}(\mu \mathrm{m})^{t, 1}$ & 16 & 1.8 & 1.0 & 125 & 0.45 \\
\hline $\mathrm{X}_{\mathrm{sa}}(\boldsymbol{\mu m})^{\mathbf{1}}$ & $\mathbf{3 . 5}$ & $\mathbf{1 . 5 5}$ & $\mathbf{1 . 0}$ & $\mathbf{2 1}$ & $\mathbf{0 . 3 9}$ \\
\hline $\mathrm{m}_{\mathrm{a}}\left(\mathrm{mg} / \mathrm{cm}^{2}\right)$ & 0.3 & 3.0 & 1.0 & 9.3 & 1.0 \\
\hline
\end{tabular}

\begin{tabular}{|c|c|c|c|c|c|}
\hline \multicolumn{6}{|c|}{ Approximation to Zero albedo $X$-ray Drive for $T \leq 200 \mathrm{eV}^{2}$} \\
\hline$\Phi_{\mathrm{R}}$ & $\mathrm{a}$ & $\mathrm{b}$ & $\mathrm{c}$ & $\alpha$ & $\beta$ \\
\hline $\mathrm{P}_{\mathrm{abl}}(\mathrm{Mbar})$ & 5.8 & 3.5 & 0 & 320 & 0.9 \\
\hline $\mathrm{X}_{\mathrm{s}}(\mu \mathrm{m})$ & 28 & 1.75 & 1.0 & 205 & 0.45 \\
\hline $\mathrm{X}_{\mathrm{sa}}(\mu \mathrm{m})^{1}$ & 5.3 & 1.45 & 1.0 & 28 & 0.36 \\
\hline
\end{tabular}

For the Hyades simulations $\mathrm{X}_{\mathrm{sa}}$ was obtained directly from the simulation and a power law then rendered rather than using the separate laws derived for $X_{s}$ and $X_{a}$.

${ }^{\dagger}$ Lindl quotes values for $P_{a b l}$ and $\mathrm{dm}_{\mathrm{a}} / \mathrm{dt}$, the $\mathrm{X}$ values are derived from these assuming $\gamma=5.3$.

${ }^{1}$ The approximate power laws for $X_{\mathrm{a}}$ and $X_{\mathrm{sa}}$ cannot be extracted easily from $\mathrm{X}_{\mathrm{s}} \sim \sqrt{ } \mathrm{P}_{\mathrm{abl}}$ and $\mathrm{dm}_{\mathrm{a}} / \mathrm{dt}$ since for $\mathrm{X}$-ray drive these scale differently with temperature. The values quoted have been obtained by approximating $\mathrm{X}_{\mathrm{s}}-\mathrm{X}_{\mathrm{a}}$ by a single temperature power law, $\mathrm{CT}^{\delta}$, by formally minimizing $\int_{1}^{2}\left[X_{s}-X_{a}-C T^{\delta}\right]^{2} d T$ with respect to $\mathrm{C}$ and $\delta$ to obtain the "best fit" coefficients. Using this approach for Hyades X-ray drive we obtain 7.95 and 1.04 for the coefficient and exponent respectively compared to the values of 7.7, and 1.0 obtained from visual inspection.

${ }^{2}$ A simple model assuming flux limited electron transport with $\mathrm{f}=.044$ gives $\mathrm{a}=26$ compared to 23.

${ }^{3}$ The values quoted are a good approximation for $T_{R} \leq 200 \mathrm{eV}$.

The 1D performance predicted by the model is intimately linked to the derived power laws, and in particular, $\mathrm{X}_{\mathrm{sa}}$, the separation between the ablation and shock fronts. It is interesting to note that for the conditions considered, $\mathrm{X}_{\mathrm{sa}}$ is not very different for any of the models, whether direct or indirect drive, although other parameters such as $\mathrm{P}_{\mathrm{abl}}$ can be quite different. This suggests that any fundamental performance advantage of one drive method over the other would derive largely from an ability to operate at higher drive flux. It is important to confirm these results with more reliable 1D simulations. 


\subsection{Target performance predictions}

Pusher thickness, $\mathrm{L}_{\mathrm{p}}$, interface travel, $\mathrm{L}_{\text {int }}$, are plotted in figure 11 as a function of laser pulse duration for direct and indirect drive. Contours of constant drive flux, derived from the Hyades power laws coupled with the results of section 2, appear as straight lines fanning out from the origin. NIF performance for various laser spots is superimposed for direct drive, and the performance predicted by the hohlraum scaling law, derived in section 3, is shown for indirect drive. To show indirect drive sensitivity, the effect of a factor 2 flux increase is also shown. These plots can be used to determine the likely target performance and parameters for a given pulse length, based on the assumptions and models described in the previous sections. It is noted that the pusher thickness is a weak function of pulse length. This is because the shock velocity scales as $\sqrt{P} \sim T^{1.8} t^{-0.12}$ and the distance traveled scales as $\mathrm{T}^{1.8} \mathrm{t}^{0.88}$ so that on the hohlraum scaling curve, the shock distance scales $\sim \mathrm{t}^{0.3}$. In fact, it can be shown that reasonably good fits to the direct and indirect drive target parameters for $5 \leq \mathrm{t}_{\text {las }} \leq 100 \mathrm{~ns}$ are

$$
\begin{array}{llll}
L_{p} & \approx 640 t_{\text {las }}^{0.22} \mu m & \\
L_{\text {int }} & \approx 540 t_{\text {las }}^{0.45} \mu m & \text { Direct drive } \\
L_{\text {exp }} & \approx 1100 t_{\text {las }}^{0.37} \mu m & \\
t_{s} & \approx 0.9 t_{\text {las }}^{0.92} & \\
L_{p} \approx 486 t_{\text {las }}^{0.18} \mu m & \\
L_{\text {int }} \approx 205 t_{\text {las }}^{0.61} \mu m & \text { Indirect drive } \\
L_{\text {exp }} \approx 524 t_{\text {las }}^{0.47} \mu m & \\
t_{s} & \approx 1.025 t_{\text {las }}^{0.87} &
\end{array}
$$

$m$

The time it takes the shock wave to reach the interface is denoted $t_{s}$ For the direct drive expressions we have applied the additional constraint that $\mathrm{L}_{\text {exp }}=\mathrm{D}_{\text {spot }}$ for reasons that will become clear shortly. These results are significant because they imply that the rate of increase of performance is a relatively weak function of the pulse length.

Superimposed on the direct drive plot of $\mathrm{L}_{\exp }$ is the line showing when $\mathrm{L}_{\exp }=\mathrm{D}_{\text {spot}}$. This is a guide to when 2D hydro effects are likely to become important. We use this condition later to make a comparison of direct and indirect drive performance. It must be stressed, however, that the practical performance achievable with either method must be quantified through careful 2D simulation and experiment.

\subsection{Comparison of model with $1 D$ hyades simulations}

In order to test the model and robustness of using the power law fits we have conducted hyades simulations for different intensities and pulse lengths. In all cases the laser and Xray drive pulses were square. NIF target dimensions have been selected from figure 11 
for a $26 \mathrm{~ns}$ laser pulse. In the direct drive system we assume $\mathrm{L}_{\mathrm{exp}}=\mathrm{D}_{\text {spot }}$ so that the appropriate intensity is $\sim 4.5 \times 10^{14} \mathrm{Wcm}^{-2}$, corresponding to an ablation pressure of about 35 Mbar when the shock reaches the interface. For indirect irradiation the achievable drive temperature is $155 \mathrm{eV}$, corresponding to an ablation pressure of around $15 \mathrm{Mbar}$ when the shock strikes the interface. In both cases, the shock reaches the interface at about 17-18 ns, and the remainder of the pulse is taken up primarily by the head of the interface rarefaction (sound wave) traveling back towards the ablation front. In order to demonstrate this optimization criterion, we have also selected target parameters for drives which end when the shock strikes the interface (ie at $26 \mathrm{~ns}$ ). According to section 2, these targets should perform less well. The results of the simulations are shown in fig 12 . R-t plots are presented in 12a for the optimized drive pulse. These clearly show the target dynamics, in particular, the period of approximately constant interface velocity followed by gradual deceleration as expected. The deceleration is more noticeable for indirect drive. In practice, the hohlraum will continue to radiate after the laser pulse, probably resulting in a reduced interface deceleration.

The model and simulated interface velocities are shown in fig 12b. The optimized drive results have been displaced vertically for clarity. However, the earlier shock arrival at the interface is real because only 17-18 ns of the 26 ns drive pulse is taken up by the shock traversing the pusher. First we note that the model does a fair job at reproducing both the magnitude of the interface velocity, and the approximately constant velocity duration, and that the optimized configuration does indeed perform better. It is also evident that the interface velocity decays weakly with time over the "constant velocity" period. This results from the weak decay of the ablation pressure with time and indicates that pulse shaping is indeed necessary to achieve exactly constant velocity. ${ }^{3}$ The significantly higher direct drive interface velocity wins over the slightly longer indirect drive constant velocity period giving direct drive better performance in agreement with the model prediction. We also note that the deceleration of the interface velocity after the rarefaction returns to the interface is less pronounced than in the ideal simulations of fig 7. This is because, unlike in those calculations, the hot ablated plasma provides a nonzero "boundary" pressure. The interface acceleration after the rarefaction kicks in at $\sim 50$ $\mathrm{ns}$ is somewhat less than $\sim-1 / 2 \mu \mathrm{m} / \mathrm{ns}^{2}$ while the interface velocity is $\sim 50 \mu \mathrm{m} / \mathrm{ns}$. Over the period to $100 \mathrm{~ns}$, vt and $\mathrm{gt}^{2}$ are therefore of the same order of magnitude so that the Rayleigh-Taylor component could be expected to be not insignificant during this time.

Finally, simulations have also been conducted for $5 \mathrm{~ns}$ pulses for a laser intensity of $10^{15}$ $\mathrm{Wcm}^{-2}$, and an X-ray drive temperature of $\sim 270 \mathrm{eV}$ to test the temporal and intensity scaling (fig $12 \mathrm{c}$ ). In these simulations the drive also ended when the shock arrived at the interface, and these are thus not optimum in the sense defined in section 2. Further the direct drive intensity is $\sim$ factor 8 below that required to make $\mathrm{L}_{\text {exp }}=\mathrm{D}_{\text {spot }}$ and would therefore be considered a very conservative design. Again, we note the model reasonably reproduces the important aspects of the simulations suggesting that the 1D model and power laws do indeed provide a good starting point for more refined design calculations.

\footnotetext{
${ }^{3}$ There are also energy flow and ionization effects that might cause variations in interface velocity during the supposedly constant velocity period. This needs to be assessed further.
} 
However, it also stresses that the accuracy of the results depends on the accuracy of the power laws derived from 1D radiation hydrodynamics simulations.

\subsection{Comparison of direct and indirect drive performance}

Target parameters are compared for each drive method in figure 13. As can be seen, direct drive promises up to $\sim$ factor 2 improvement over indirect drive for short pulses, reducing to less than 50\% improvement above $~ 35 \mathrm{~ns}$ (see fig 15). The main reason for this is the ability of direct drive to operate at high intensity within the assumptions of the model. The on target flux/intensity is shown in figure 14 together with the pressure generation as a function of pulse length. The laser intensity on target is much larger ( factor 10) than the X-ray flux particularly at shorter pulses, and the ablation pressure is correspondingly higher for direct drive (up to $\sim$ factor 3 ). This needs to be verified with more reliable $1 \mathrm{D}$ simulations.

The importance of the ability to control 2D effects for direct drive can be seen in figure 15 where we show the ratio of the width of the drive area (hohlraum diameter, or focal spot size) to the length of the experiment. For direct drive we have made this unity. However, we see that $\mathrm{L}_{\mathrm{exp}} / \mathrm{D}_{\text {driver }} \leq 0.3$ for indirect drive indicating that $2 \mathrm{D}$ effects are unlikely to be a limiting factor for X-ray driven targets. To quantify this, if in practice it is necessary to operate laser driven systems such that $\mathrm{D}_{\text {spot }} \approx 2 \mathrm{~L}_{\text {exp }}$, any significant advantage over indirect drive is lost (see fig 13). On the other hand, if we can successfully control 2D effects by suitable tamping and irradiation geometry, then direct drive may do even better.

\subsection{Effect of the number of beams / available energy}

From section 3 it is seen that the X-ray drive temperature is a weak function of the available energy. This is because as less energy is fed into the hohlraum we can afford to make it smaller from a filling point of view, thereby reducing the wall losses and recovering some of the energy available to drive a target. In the case of direct drive, as the laser energy is reduced the experimental length also decreases rather slowly. If we preserve the ratio of $\mathrm{L}_{\text {exp }} / \mathrm{D}_{\text {spot }}$ we can also afford to decrease the spot diameter, thereby recovering the intensity somewhat. These effects lead to a weak dependence of target performance on the available laser energy. This is shown in figure 16 where target parameters are plotted for 96,48 , and 24 NIF beams. The performance for 24 beams is predicted to be about $80 \%$ of that at full energy. This is very significant because it implies great flexibility, leaving many beams for multiple backlighters, and suggests that valuable experiments can be performed early in the commissioning of NIF as long as the beam geometry can provide adequate symmetry. Finally it is noted that the relative performance is virtually unaffected by the available energy.

\subsection{A comment on pressure regimes and target structure}

So far we have not considered the state of the shocked materials. A detailed discussion of the influence of material properties and energy flow on the development of instabilities 
is far beyond the scope this work. Here we simply raise this as an issue that should be considered. There are two extremes to be addressed. On the one hand at short pulses, $\geq$ 100 Mbar shocks are promised. This corresponds to temperatures $\sim 50 \mathrm{eV}$ in the $\mathrm{CH}$ pusher, sufficient to form a small radiative precursor. In the foam, temperatures of $\sim 100$ $\mathrm{eV}$ would result from shock heating if radiation effects were ignored, which of course they cannot be under these circumstances, and we conclude that radiation effects must be significant in these targets. If we could operate direct drive at close to $10^{16} \mathrm{Wcm}^{-2}$, which is close to optimized in the sense $\mathrm{L}_{\text {exp }} \approx \mathrm{D}_{\text {spot }}$ for a $\sim 5$ ns pulse, without laser plasma instabilities dominating the experiment, we would expect to generate pressures close to $400 \mathrm{Mbar}$ in the pusher, and $100 \mathrm{Mbar}$ in the foam. This would clearly open new regimes for a variety of experiments.

At the other end of the scale we generate $\sim 10$ Mbar pusher shocks for long pulses and few Mbars in the foam. This still corresponds to $\sim 10 \mathrm{eV}$ foam temperatures, well into the plasma regime. However, at the lower drive pressures (longer pulses) the foam structure may become more influential on the hydrodynamics, in seeding instabilities, and on the growth itself, particularly as there would be less radiative preheating from the shocked material to help homogenize the foam ahead of the shock wave. While these effects may turn out to be small they certainly must be considered, particularly as many experiments rely on using low density foam targets. We need both experiment and theory to support studies in this area. 


\subsection{Mach Number Considerations}

An important parameter in the development of shock driven instabilities is the Mach number, $\mathrm{M}$, of the incident shock wave (defined as the shock speed in units of the sound speed in the up-stream material, ahead of the shock). This is because the Mach number determines how compressible the interaction is. It is straightforward to show that the compression induced by a shock wave in a fluid with constant specific heat ratio, $\gamma$, is

$$
\frac{\rho_{2}}{\rho_{1}} \quad=\frac{M^{2}(\gamma+1)}{M^{2}(\gamma-1)+2} \Rightarrow \frac{(\gamma+1)}{(\gamma-1)}, M \geq 5
$$

where 1 and 2 refer to pre and post shock states respectively. For typical laboratory plasmas $\gamma \sim 1.5$, and the above ratio asymptotes towards $(\gamma+1) /(\gamma-1)$ approximately for $\mathrm{M}$ $\geq 5$. Lasers can generate much higher pressures than other laboratory drivers, and are therefore attractive for accessing the "fully" compressible regime. A critical consideration for laser drivers, however, is preheat from X-rays and fast electrons. This is because, raising the temperature ahead of the shock increases the sound speed, requiring a larger shock velocity, and therefore drive pressure, to maintain the Mach number. Below we begin to address how to assess achievable regimes with laser drivers. Recognizing that preheat is a governing parameter, and to some extent controllable, we take the approach of determining the level of preheat allowable to achieve a Mach number up to a given value. The target design must then take this into account.

\subsection{Relating Mach number to drive pressure}

The Mach number is determined by the ratio, $\mathrm{p}$, of the shock (drive) pressure to that in the pre-shocked (preheated) material ${ }^{4}$

$$
M^{2}=\frac{\left(\gamma_{1}-1\right)}{\left(\gamma_{2}-1\right)} \frac{1}{2 \gamma_{1}}\left\{\left(\gamma_{2}-1\right)+\left(\gamma_{2}+1\right) p\right\} \frac{1-p}{1-\frac{\left(\gamma_{1}-1\right)}{\left(\gamma_{2}-1\right)} p}
$$

For $\gamma_{1}=\gamma_{2}=\gamma$ this reduces to

$$
M^{2}=\frac{1}{2 \gamma}\{(\gamma-1)+(\gamma+1) p\}
$$

For a typical $\mathrm{CH}$ pusher plasma, and for $\mathrm{p} \geq 2$, a good approximation is $\mathrm{M} \approx 0.9 \mathrm{p}^{1 / 2}$. The simplified expression above is displayed in figure 17 for various $\gamma$. As can be seen, $\gamma$ has only a small effect on the Mach number for a given pressure ratio. These results can be used to estimate drive pressure as a function of preheat pressure (and temperature through the EOS), and Mach number. For example, it can be seen that to achieve $M \approx(3,5,10)$ requires $p \approx(10,30,120)$. Figure 18 includes preheat pressure against

\footnotetext{
${ }^{4}$ For this expression to be valid requires the following approximations to hold: $\mathrm{c}_{\mathrm{s} 1} \approx(\gamma \mathrm{P} / \rho)_{1}{ }^{1 / 2}$; $\mathrm{h}_{1,2}=\{\gamma /(\gamma-1) \mathrm{P} / \rho\}_{1,2}$. The sound speed $\left(\mathrm{c}_{\mathrm{s} 1}\right) \gamma$, refers only to the term $1 / 2 \gamma_{1}$
} 
temperature calculated from hyades EOS data, and the corresponding drive pressure curves for $M \approx(3,5,10)$. These results depend on the validity of the simplifying EOS approximations leading to the above expressions. Superimposed on the plot is the sound speed calculated by hyades and a perfect gas type sound speed with $\gamma=1.5$, showing that at least this part of the approximation is reasonable. The Mach numbers were also checked using the same $\gamma$, and it was found that $\mathrm{p} \approx 130$ was a slightly better match for $\mathrm{M}=10$, and this is the ratio plotted, rather than 120 . These should be verified with hydrocode simulations, which could also be used to construct such plots for a variety of potential pusher materials, using the above expressions as a guide.

\subsection{Direct drive performance}

The drive pressure generated by direct drive is also shown as a function of laser intensity in figure 18. As the laser intensity, and therefore drive pressure, is increased, the allowable preheat to achieve say $M \geq 5$ also increases. From previous discussion we know it is possible to operate at $\mathrm{I}_{\text {las }} \sim 3 \mathrm{X} 10^{15} \mathrm{Wcm}^{-2}$ over a pulse length between 5 and10 ns and keep $\mathrm{L}_{\mathrm{exp}} \approx \mathrm{D}_{\text {spot}}$. Provided adequate interface planarity is maintained, figure 18 indicates that the preheat temperature must remain below $\sim 10-15 \mathrm{eV}$, which should be easily achievable. If we needed to operate at $\sim 10^{14} \mathrm{Wcm}^{-2}$ then we would need to keep preheating below about $1 \mathrm{eV}$, which also seems feasible, particularly given pusher thicknesses.

If it is assumed that $\mathrm{L}_{\text {exp }} \approx \mathrm{D}_{\text {spot }}$ is the appropriate optimization parameter, operating at $10^{14} \mathrm{Wcm}^{-2}$, and $\sim 60 \mathrm{~ns}$ gains roughly a factor 4 over the comparable point at $3 \times 10^{15}$ $\mathrm{Wcm}^{-2}$ and $\sim 8 \mathrm{~ns}$. If it is the case that lower pressures result in better planarity, then this factor might increase. Thus provided preheat, and planarity can be adequately controlled, long pulses of many tens of ns remain attractive.

Simulations are necessary to bound preheat levels and to demonstrate that adequate control is feasible within the objectives of the experiment. Experimental methods to confirm preheat bounds either directly or indirectly will be necessary. Consideration should be given to assessing the role of hot electrons in preheating.

\subsection{Indirect drive performance}

The above analysis has been repeated for X-ray driven targets. The results are shown in figure 19. The X-ray flux achievable is determined by the hohlraum performance.

Smaller hohlraums will increase the flux but at the risk of greater preheating both from $\mathrm{X}$-rays, and hot electrons. Reducing preheat to $\mathrm{eV}$ levels in $\mathrm{X}$-ray driven targets is more demanding than for directly driven systems because of the copious quantities of hard Xrays and their relatively long mean-free-path, $\mathrm{mfp}$, in low $\mathrm{Z}$ materials. For example, the mfp of $2.5 \mathrm{keV}$, gold M-band X-rays in solid CH is $\sim 60 \mu \mathrm{m}$, and typically $\sim 10 \%$ of the laser energy can be converted into such X-rays. Without efforts to control preheat, thin, undoped pushers are easily heated to $\sim 10 \mathrm{eV}$ in $1 \mathrm{~ns}, \mathrm{~T}_{\mathrm{R}} \sim 200 \mathrm{eV}$ Nova experiments. However, very much thicker targets will be used on NIF $(\sim 10-20 \mathrm{mfp}$ for $2.5 \mathrm{keV}$ 
photons), and while we anticipate some design effort will be necessary, controlling preheat to $\mathrm{eV}$ levels is not expected to be problematic.

It is expected that NIF will typically operate in the $t \geq 5 \mathrm{~ns}$ range and correspondingly $T_{R}$ $\leq 260 \mathrm{eV}$. To achieve $\mathrm{M} \geq 5$ under these conditions, preheat must be kept below $\sim 7 \mathrm{eV}$, which is probably achievable. For comparison, this is roughly half the preheat temperature allowable for direct drive at a comparable pulse length. The $1 \mathrm{eV}$ preheat constraint occurs at about $155 \mathrm{eV}$, and $\sim 26 \mathrm{~ns}$ if $\mathrm{M} \geq 5$ is required.

As with direct drive, simulations are necessary to more accurately assess preheat levels and necessary control measures. These might impact on the effective performance.

\subsection{Discussion}

In the experiments considered here, lower pressures are generated at comparable pulse lengths with indirect drive compared to direct drive because of the energy "lost" in heating the hohlraum. As a consequence, the upper preheat temperature allowable is lower for X-ray drive than for laser drive (at comparable pulse lengths) by about a factor 2. Without more information on actual preheating levels, it is difficult to draw concrete conclusions. However, if we select $1 \mathrm{eV}$ as a lower limit on our ability to control preheat, then we must operate above approximately $2 \mathrm{X}_{10}{ }^{14} \mathrm{Wcm}^{-2}(\mathrm{t} \leq 50 \mathrm{~ns})$ for direct drive, and above $\sim 155 \mathrm{eV}$ ( $\mathrm{t} \leq 26 \mathrm{~ns}$ ) for indirect drive to achieve $\mathrm{M} \geq 5$. Under these circumstances, direct drive's advantage over indirect drive is exaggerated.

It is possible to quantify the relative performance further if it is assumed preheat is either negligible or the same for both drive methods. Then Mach number is synonymous with ablation pressure. This is quantified in fig 20 in which target performance ratio is plotted applying the additional constraint that both drive methods operate at the same drive pressure. We have chosen to plot this against direct drive pulse length, displaying the corresponding, shorter indirect drive pulse length as a ratio also. For comparison we have included the performance ratio for operating at the same pulse length (as opposed to ablation pressure). As can be seen, if we require that both methods operate at the same ablation pressure, then direct drive can in principle use longer pulses, and make further gains over indirect drive.

Naively it is expected that we will need greater efforts to control preheat in X-ray driven targets than their directly driven counterparts, particularly for shorter pulse lengths. On the other hand pushers are relatively thick, and generating significant preheat may turn out to be challenging, even requiring separate hard X-ray sources. This is an additional advantage of the single sided design particularly for experiments in which significant preheat is required.

Finally, these results are dependent on the accuracy of the EOS data used, and should be regenerated with the most up to date data, and for various potential pusher materials. 


\subsection{Conclusions}

We have developed a simple understanding and model to describe the 1D hydrodynamics of laser and X-ray irradiated, planar targets, consisting of a pusher acting on a low density foam, driven by steady shocks. The model accurately predicts the propagation of hydrodynamic waves in targets and in particular predicts the distance the pusher-foam interface can be driven under conditions of constant velocity. This is particularly relevant for, amongst others, Richtmyer-Meshkov and equation of state studies. The distance the interface travels at constant velocity is shown to be a simple multiple of the effective shock-ablation front separation, the denstiy ratio between the unshocked foam and pusher, and to a lesser extent on the EOSs of the components. For a given drive this distance is maximized by driving the target until the rarefaction reflected from the interface upon shock arrival reaches the ablation front.

It appears that for NIF direct drive out-performs indirect drive by up to $\sim$ factor 2 for short $\sim 5 \mathrm{~ns}$ pulses at the same pulse length, and typically $\sim$ factor 2 at the same ablation pressure, which is important for Mach number scaling. For a $\sim 1 \mathrm{~g} / \mathrm{cc}$ pusher and $0.1 \mathrm{~g} / \mathrm{cc}$ foam constant velocity interface travel is about $1 \mathrm{~mm}$ at 5-10 ns rising to 3-4 mm close to $100 \mathrm{~ns}$. Pusher thicknesses are typically $\sim 1 \mathrm{~mm}$ and in the case of direct drive laser intensities are moderate accept at the shortest pulses so that with modern smoothing techniques filamentation is probably controllable, but this needs to be verified. These results are only weakly dependent on the available energy, with the interface travel falling to $\sim 80 \%$ of its original value if the number of beams is reduced by 4 from 96 to 24.

The validity of these predictions relies on the accuracy of the 1D hydrodynamics modeling used to predict the shock-ablation front separation, as well as the assumed drive performance. The 1D modeling needs to be corroborated with a more reliable computational model. For indirect drive a temperature scaling was normalized to lasnex simulations at $3 \mathrm{~ns}$ and was used to extrapolate to many $10 \mathrm{~s}$ ns based on a constant filling model. While this is likely to be a good approximation of achievable performance, it is not necessarily optimum. Several careful 2D simulation could easily bound likely performance and refine the quantitative results. For direct drive the critical factor is the ability to control 2D effects of laser-target coupling and hydrodynamics. The above conclusions concerning performance assume it will be possible to maintain adequate interface planarity over an experimental length of 1 focal spot diameter, based on lateral relaxation times. If a practical limit turns out to be $1 / 2$ focal spot diameter, then the performance of both methods is roughly equal. Although 2D simulations would probably do well at quantifying 2D hydro effects and showing how to mitigate them, experiments will undoubtedly be required to verify laser target coupling predictions. An additional untested element for both methods is the very long pulse regime. Any problems associated with using many 10s ns pulses will only be uncovered as experiment begin.

The performance of the target in terms of the constant velocity interface travel is a weak function of the pulse length $\sim \mathrm{t}^{1 / 2}$, but long, many $10 \mathrm{~s}$ ns pulses remain attractive. A constraint is imposed on the lower intensity or drive temperature, and therefore pulse 
length, usable by the Mach number required for the pusher shock. The lower limit will depend on the actual preheat levels in the targets, and these will need to be assessed. At $1 \mathrm{eV}$ preheat in a $\mathrm{CH}$ pusher, the intensity and temperature limits are $\sim 2 \mathrm{X} 10^{14} \mathrm{Wcm}^{-2}$ $(\sim 60 \mathrm{~ns})$ and $\sim 155 \mathrm{eV}(\sim 26 \mathrm{~ns})$ respectively.

Many experiment advocate or indeed necessitate the use of low density foam components. While it has been recognized that the foam structure might influence dynamics this has largely been glossed over partly because only recently has it become feasible to begin to predict foam structure effects with the advent of new methods and computers. Anomalies are often observed in experiments involving foam components. It is therefore important to invest both experimental and computational effort to ensure that foams are adequately understood to ensure that experiments are correctly interpreted.

Finally, it is noted that at short, $\sim 5 \mathrm{~ns}$ pulses, NIF is capable of illuminating targets with intensities $\sim 10^{16} \mathrm{Wcm}^{-2}$, generating $\sim 400$ Mbar pressures over $\sim 1 \mathrm{~mm}$ scale focal spots. This would open up exciting possibilities, but may not be feasible because of laser plasma instabilities. The physics potential warrants estimates of the ability of modern techniques to control these instabilities to an acceptable level.

This work was performed under the auspices of the U.S. Department of Energy by the University of California Lawrence Livermore National Laboratory under contract No. W7405-Eng-48. 


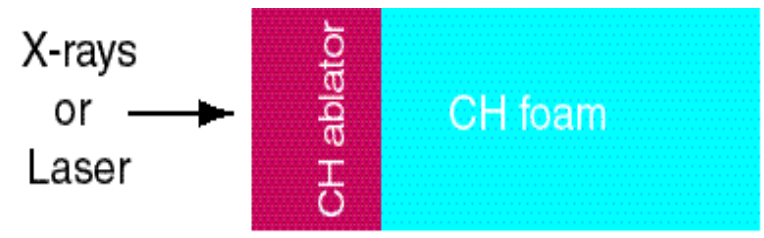

Figure 1 Schematic of generic target consisting of a high density pusher and low density foam

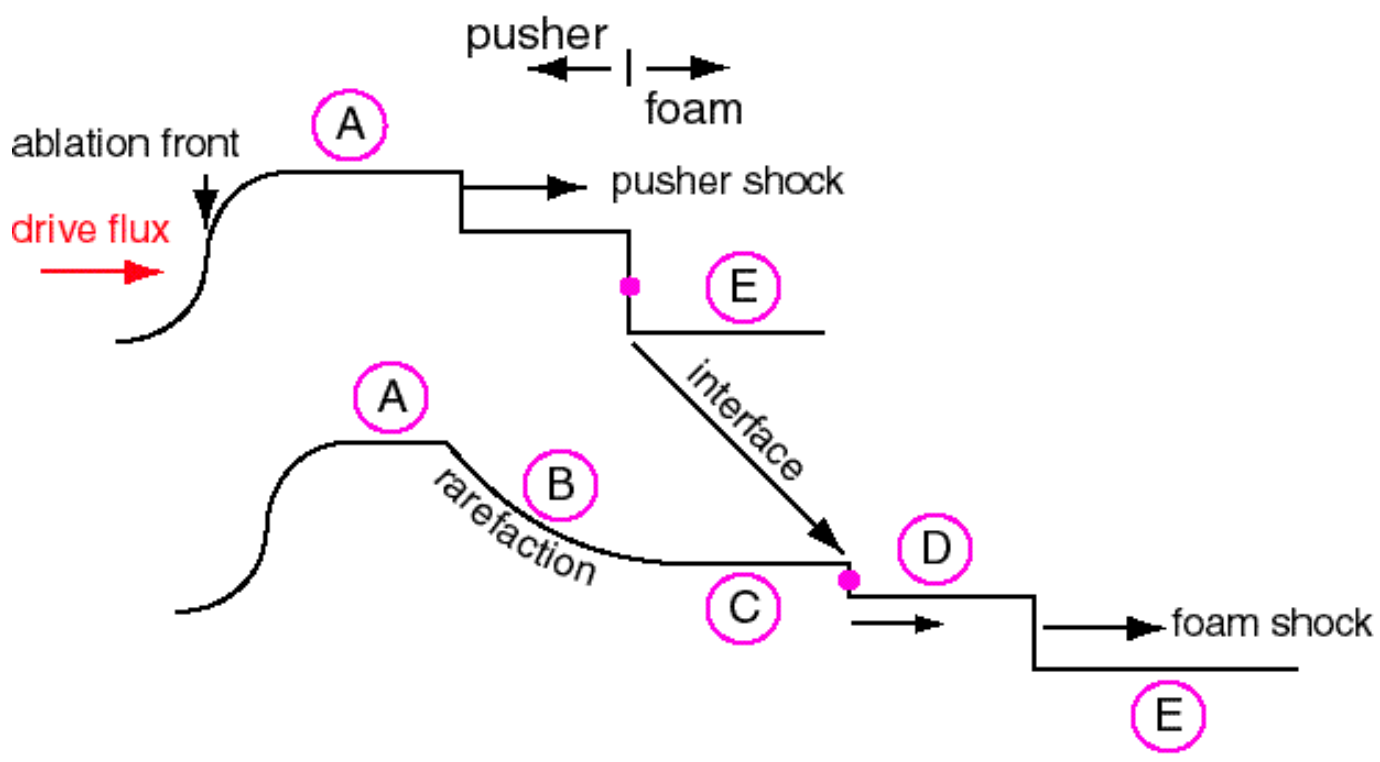

Figure 2 Schematic of target dynamics before and after the shock wave has passed through the interface: A) shocked pusher; B) rarefaction wave; C) constant velocity region connecting rarefaction to interface; D) shocked foam; E) unshocked foam. In reality energy flow (due primarily to radiation for the conditions considered) causes departures from the ideal profiles depicted above. 


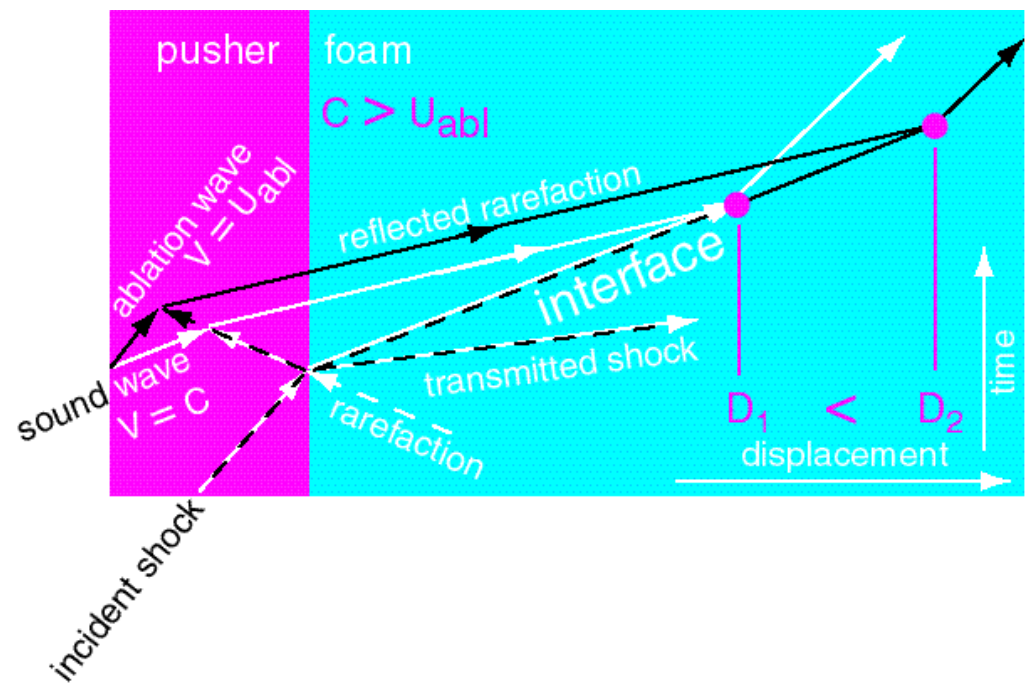

Figure 3 Schematic of wave dynamics in the target. The black lines show the situation in which the drive (ie an ablation wave) is maintained until the interface rarefaction reaches the ablation front, while the white lines depict the bahvior if the drive is turned off when the shock wave reaches the interface.

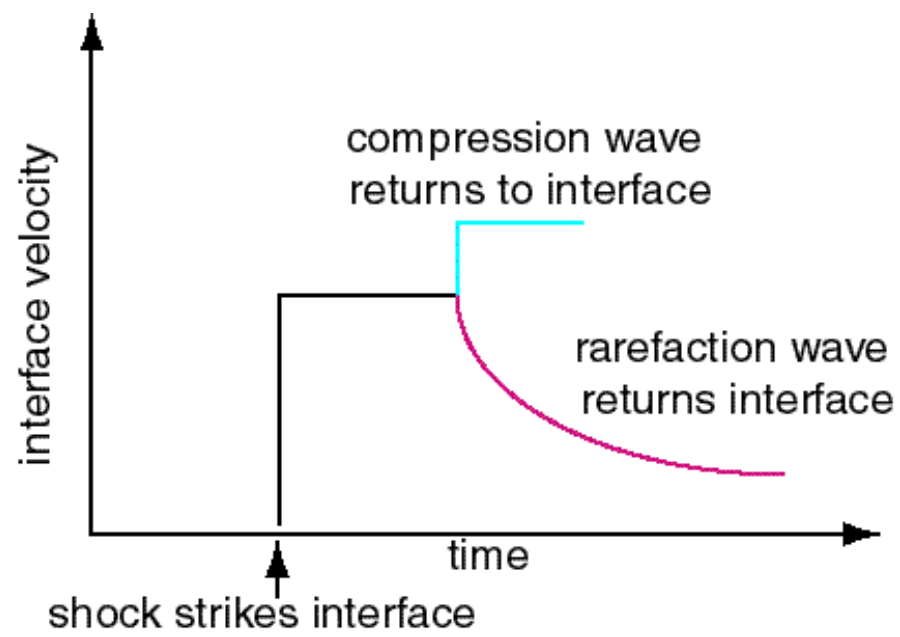

Figure 4 Schematic of interface velocity history showing two possible scenarios depending on the drive history. A compression returns to the interface if the drive is maintained after the interface rarefaction reaches the ablation front. 


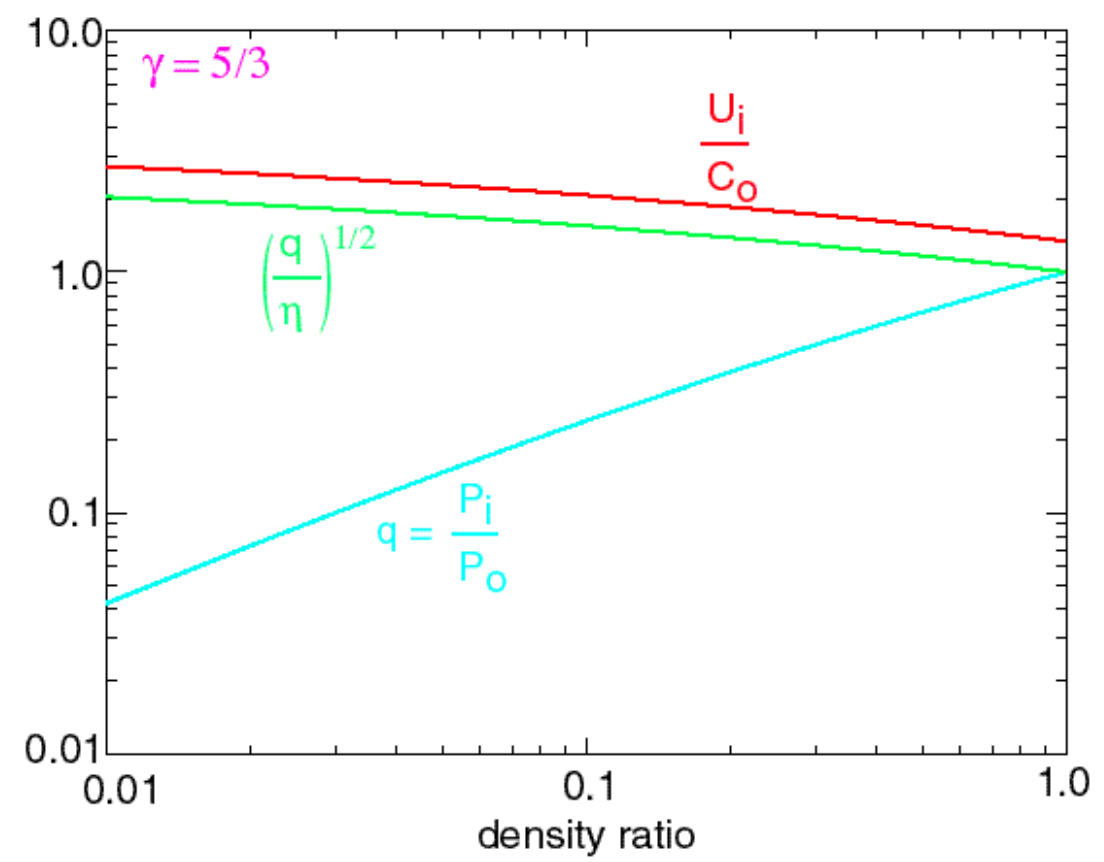

Figure 5 Solutions for a strong shock passing through a step down interface. Both materials have $\gamma=\mathbf{5 / 3}$.

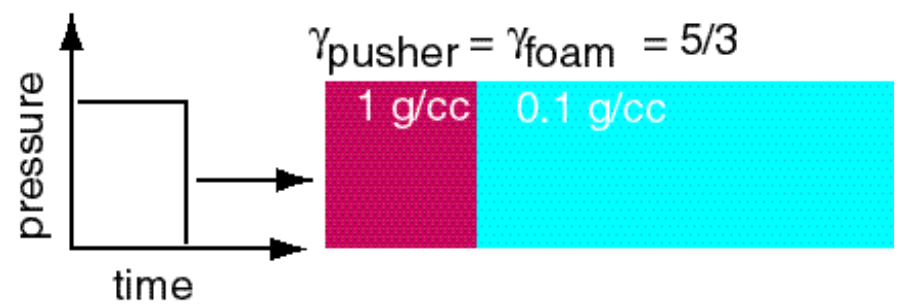

Figure 6 Schematic of set up for 1D idealized simulations to test the 1D target dynamics model. The pressure pulse ends when the interface rarefaction reaches the left edge of the pusher. In reality the pressure does not drop instantaneously to zero when the drive is turned off. This results in a weaker decay of the system, and a reduced deceleration of the interface when the rarefaction eventually returns. 

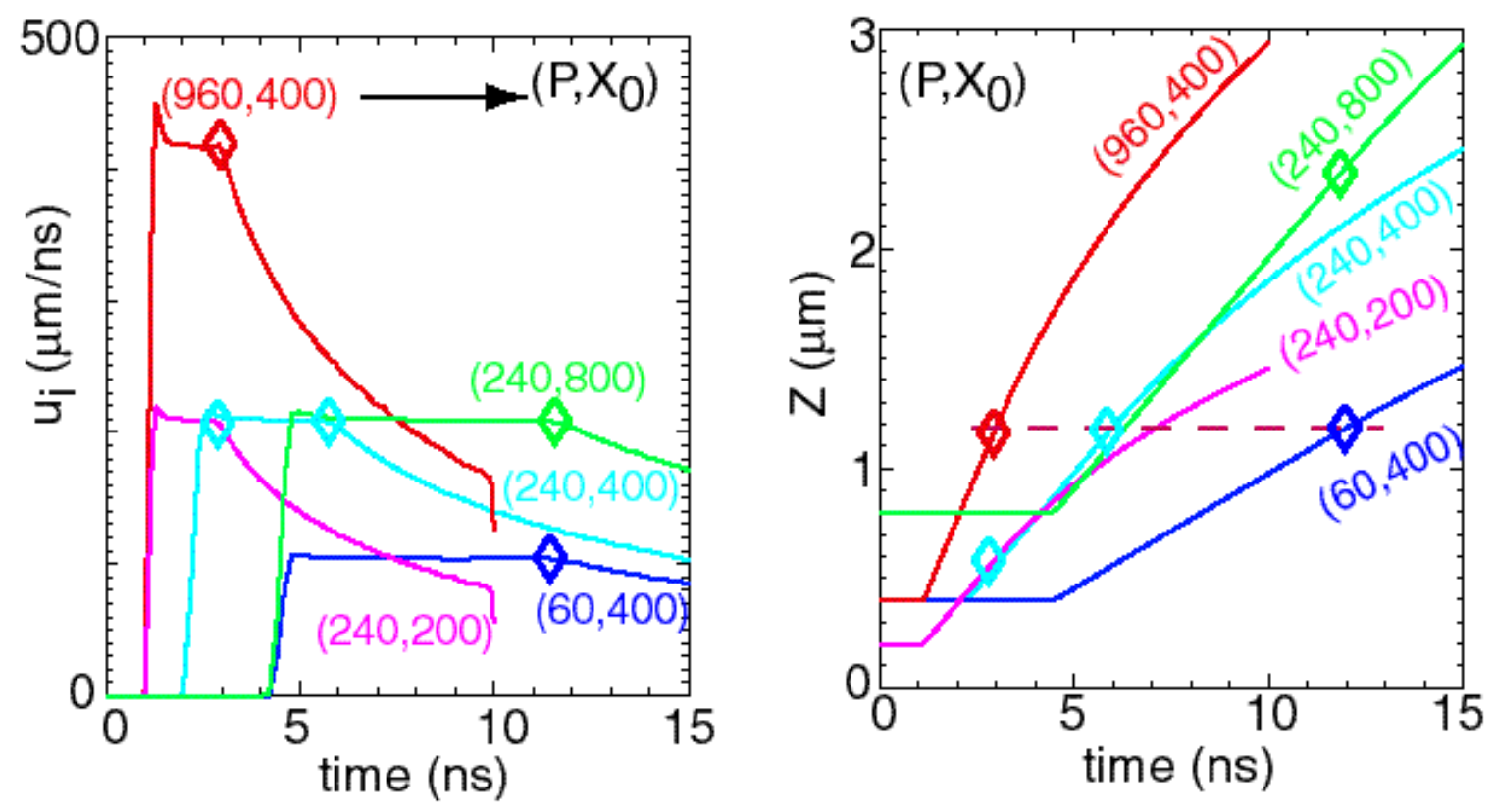

Figure 7 Interface velocity history (left) and trajectory (right) predicted by hyades for various initial configurations labeled by drive pressure and pusher thickness $\left(P, X_{0}\right)$. The results confirm the prediction of the $1 D$ hydrodynamics that the distance traveled by the interface under constant velocity conditions depends only on the width of the compressed pusher (for given target compositions). There is also likely to be a weak pressure dependence because of sound speed variations. This will also be material dependent).

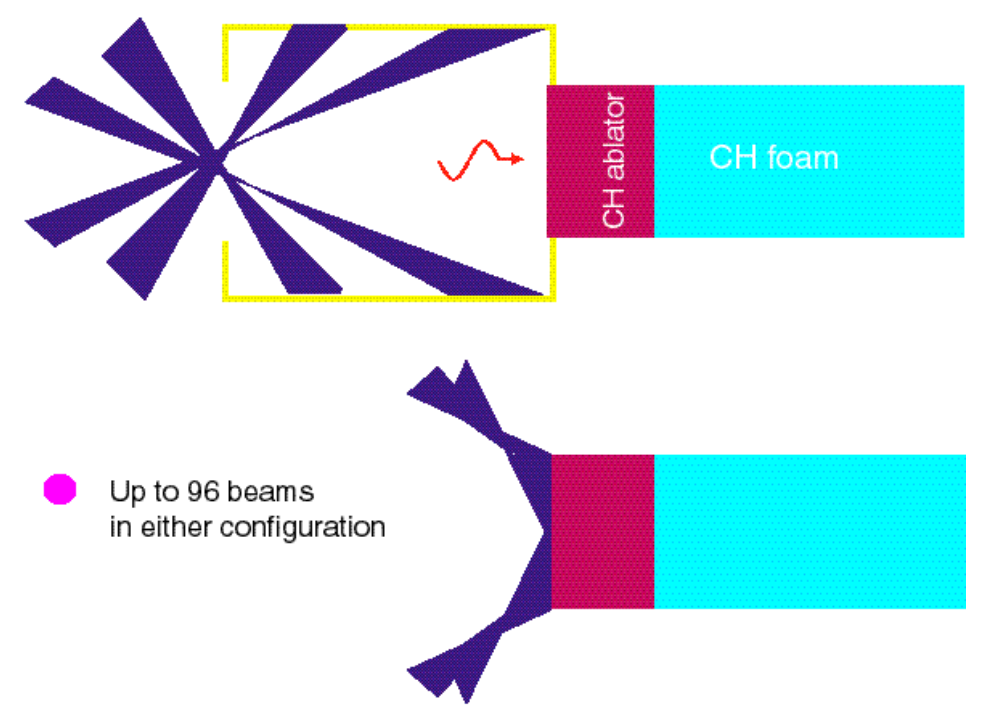

Figure 8 Schematic of drive configurations considered for NIF. 


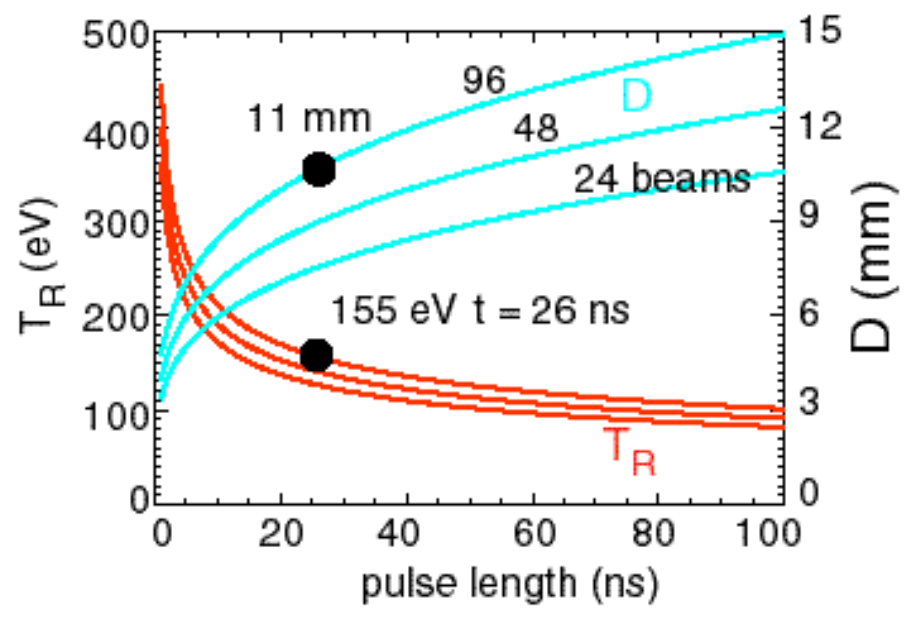

Figure 9 Drive temperature and hohlraum size predicted by the energy balance model.

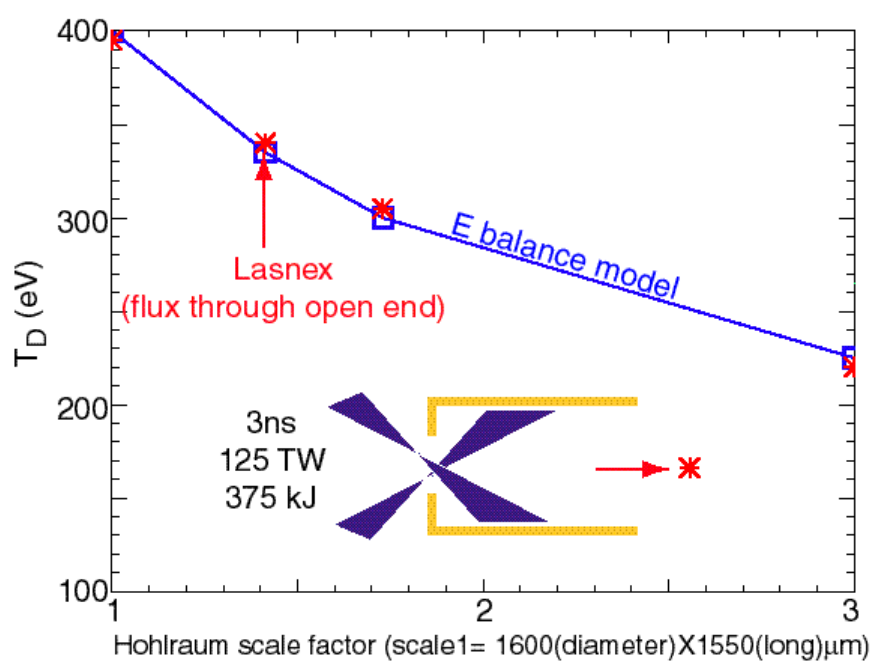

Figure 10 Comparison of Energy balance model made to fit Lasnex simulations of the effective drive temperature from the open end of a half hohlraums driven with $375 \mathrm{~kJ}$ of $3 \omega$ laser energy for $3 \mathrm{~ns}$. 


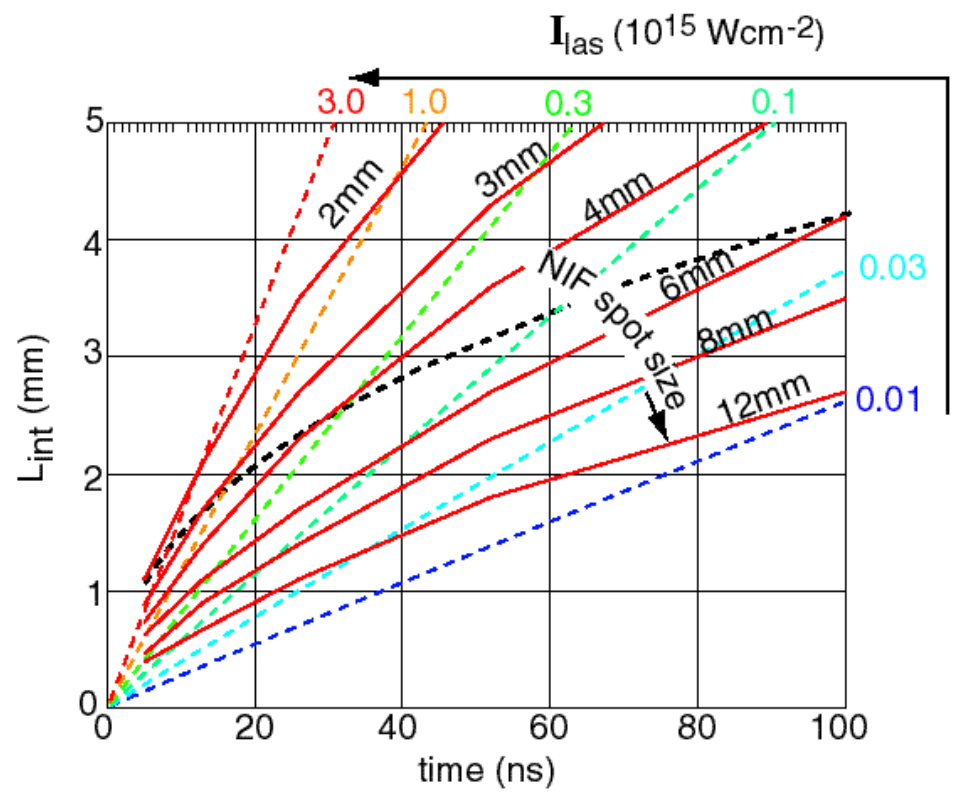

Figure 11a Constant velocity interface travel as a function of pulse length predicted for direct drive. Lines of constant intensity are shown as dashed lines fanning out from the origin. NIF performance is overlaid as solid red lines for various focal spot sizes. The dashed black curve is predicted performance for the condition $L_{\text {exp }}=D_{\text {spot}}$ •

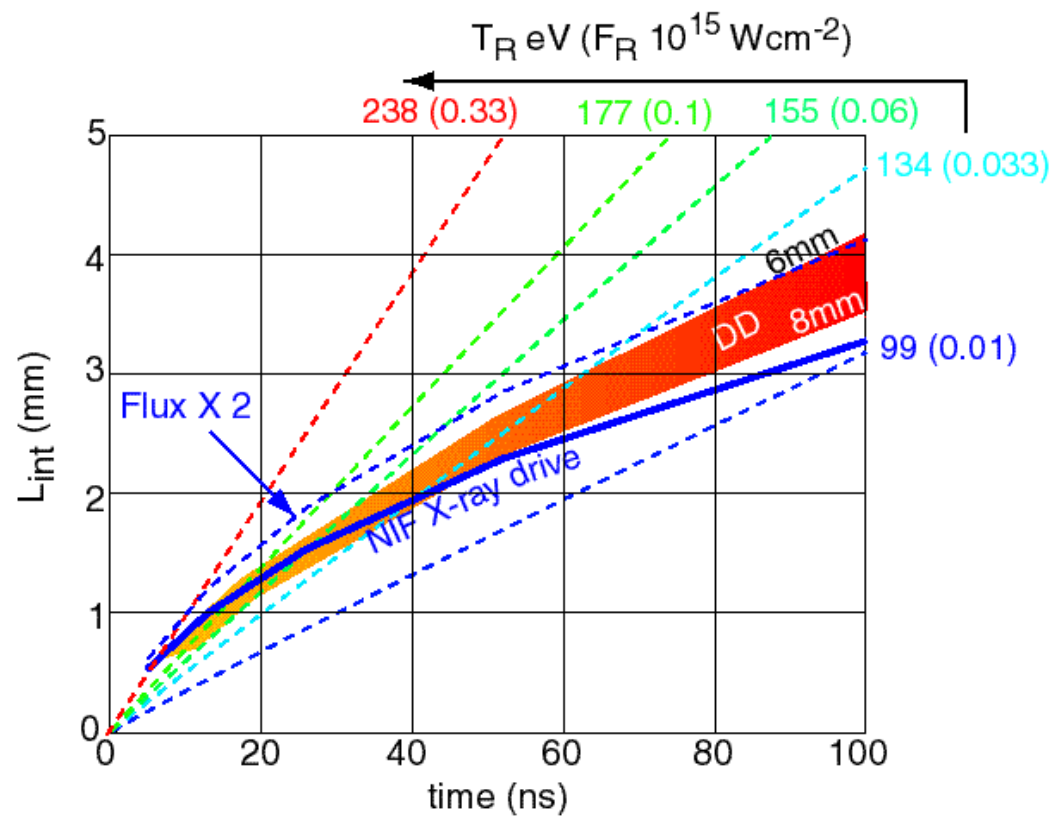

Figure 11b Constant velocity interface travel as a function of pulse length predicted for indirect drive. Lines of constant flux are shown as dashed lines fanning out from the origin. NIF performance is overlaid as a solid blue line. A factor of 2 increase in drive flux is depicted by the dashed blue curve indicating the sensitivity to the drive model. The direct drive region bounded by 6 and $8 \mathrm{~mm}$ focal spots is also shown for comparison. 

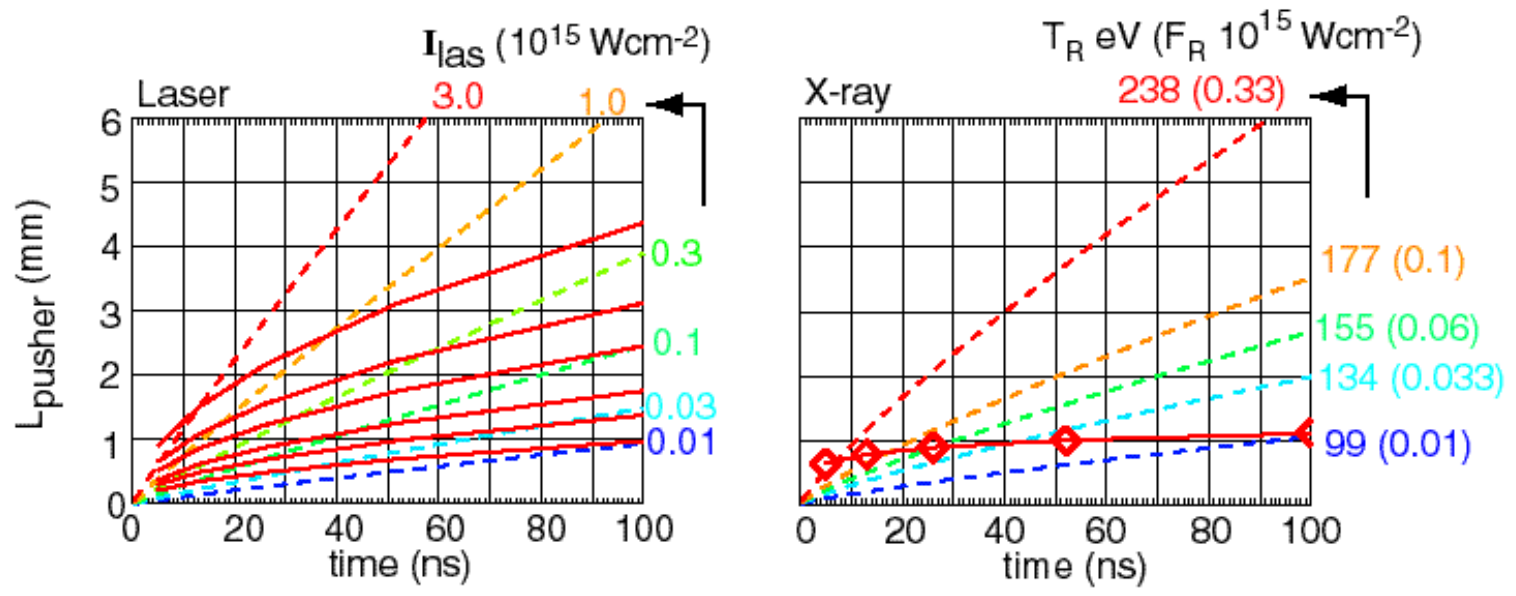

Figure 11c Model predictions of $L_{p}$ for direct and indirect drive. The labeling is similar to figure 11a\&b. The length of the experiment is just $L_{\text {exp }}=L_{p}+L_{\text {int }}$.
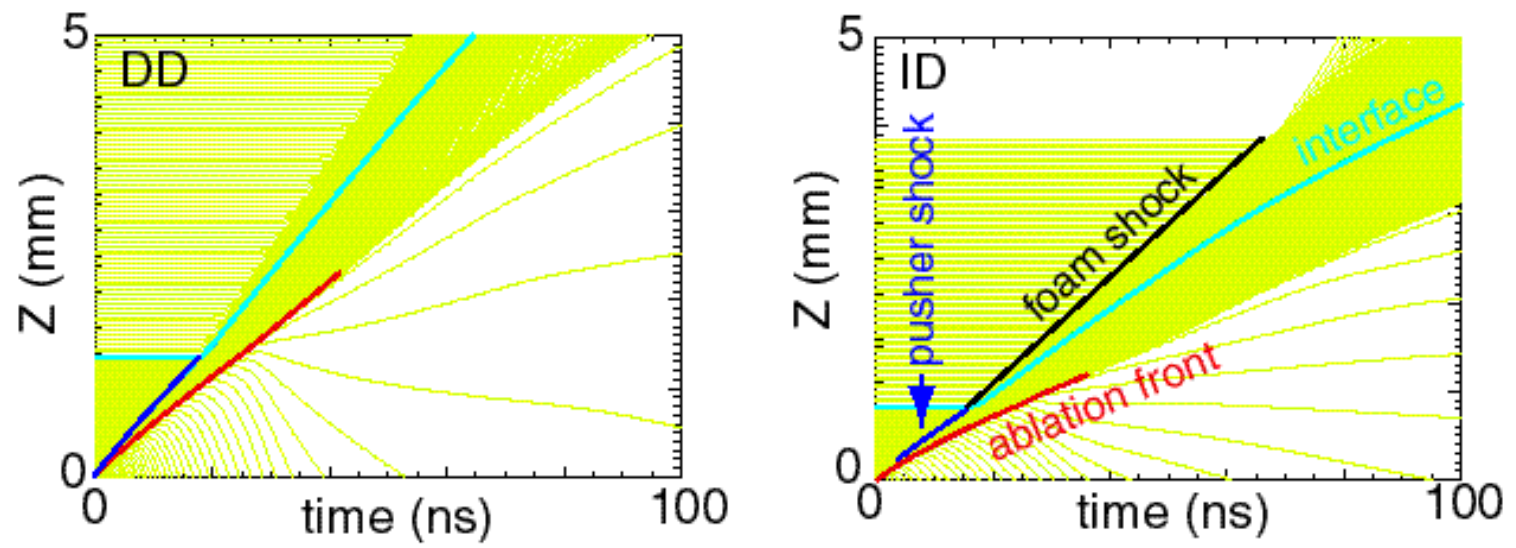

Figure 12a Rt plots showing dynamics for direct drive (left) and indirect drive (right) for a laser intensity of $4.5 \times 10^{14} \mathrm{Wcm}^{-2}$ and $\mathrm{X}$-ray drive temperature of 155 $\mathrm{eV}$ respectively. The pulses were square and $26 \mathrm{~ns}$ duration in both cases. 


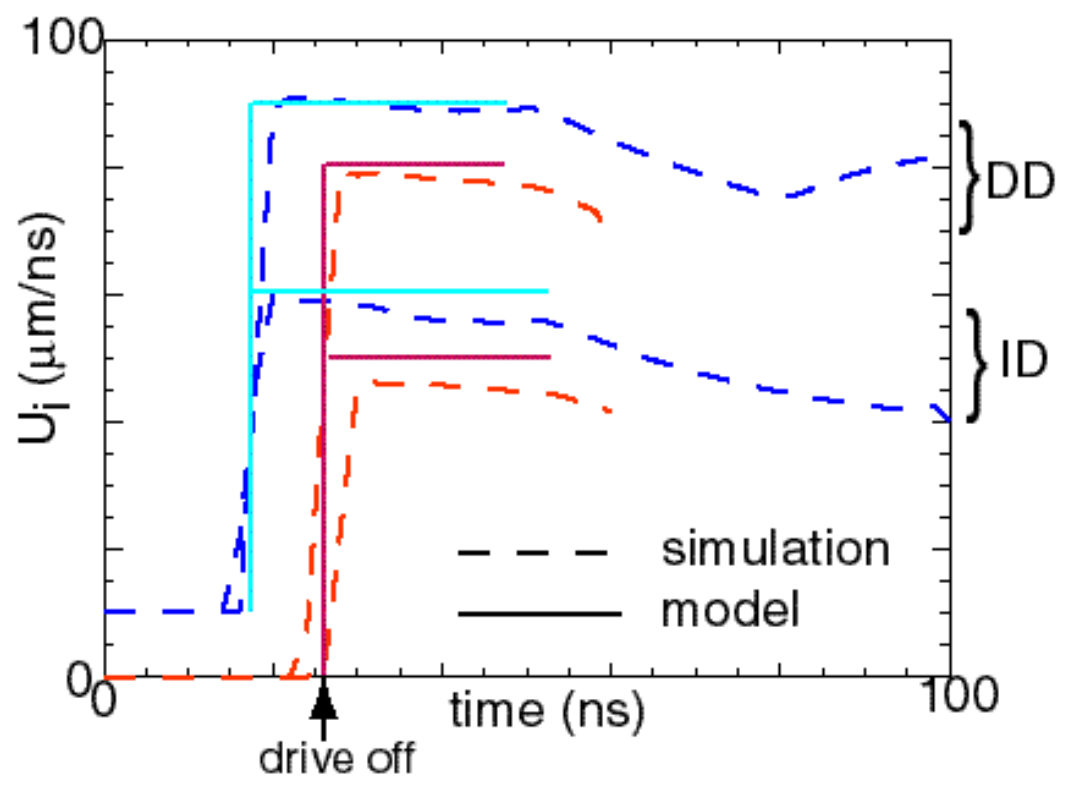

Figure 12b Comparison of model predictions with hyades simulations for optimized drive-target configurations also shown in fig 12 a (blue), and for a less efficient target in which the drive ends when the shock strikes the interface (red). The blue curves have been displaced vertically by $+10 \mu \mathrm{m} / \mathrm{ns}$ for clarity.

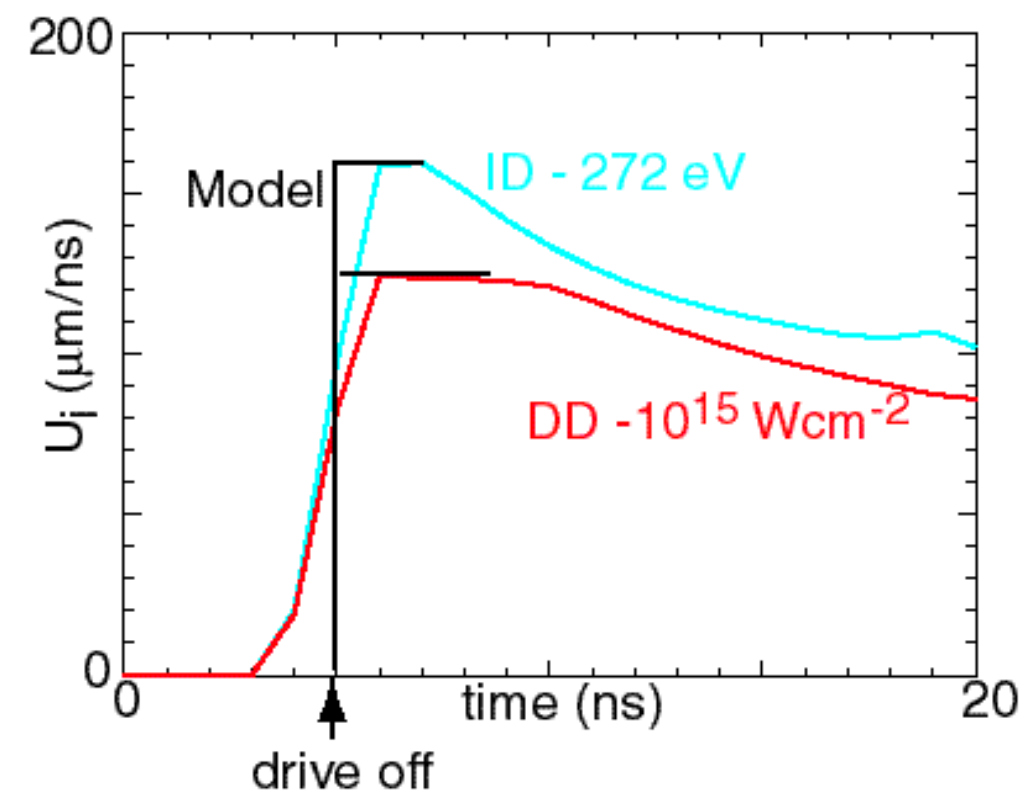

Figure 12c Model predictions compared with simulations for short, high drive targets. The apparently slow rise in the interface velocity is a numerical artifact and due to the spatial resolution of the calculation and the temporal sampling of the result. 


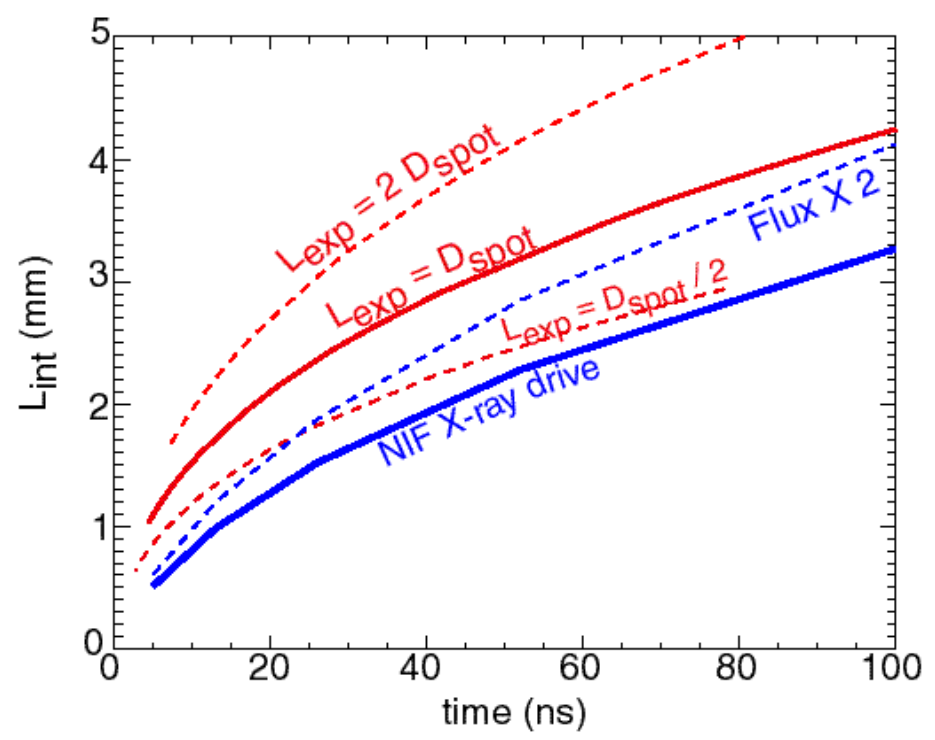

Figure 13 Comparison of direct and indirect drive performance predicted for NIF in terms of constant velocity interface travel. The direct drive is potentially up to factor 2 better than indirect drive, but performance depends critically on the ability to control 2D effects.
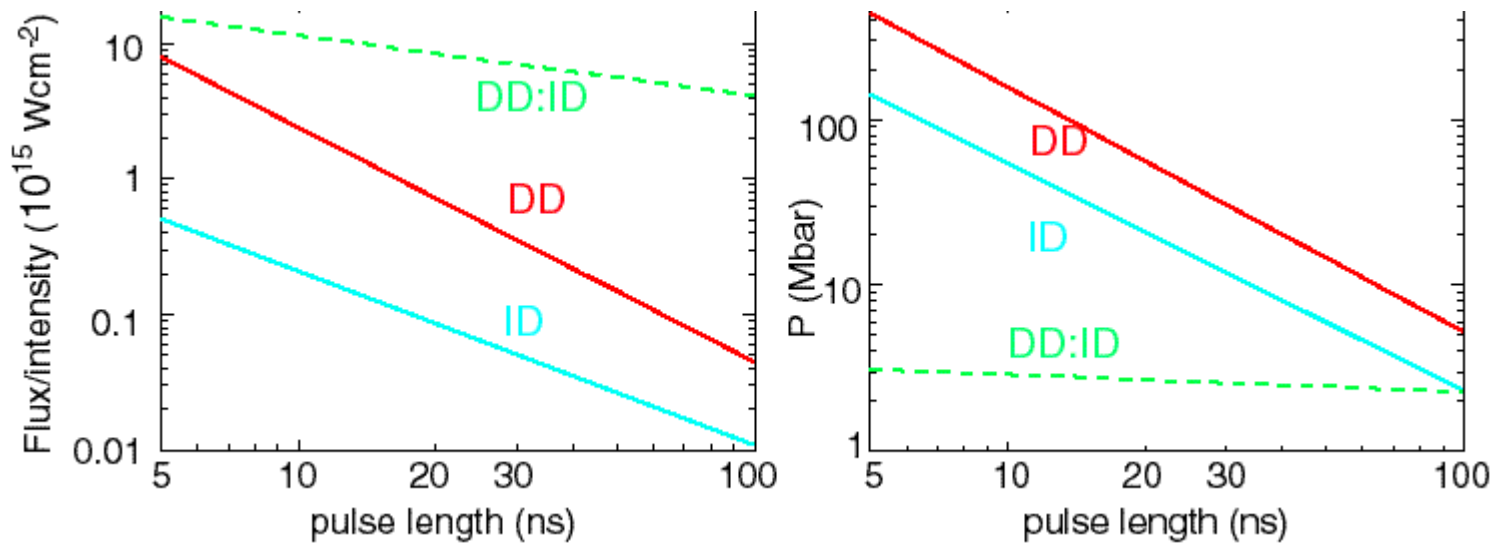

Figure 14 Comparison of flux on target and ablation pressure for direct and indirect drive predicted for NIF assuming $L_{\text {exp }}=D_{\text {spot }}$ for direct drive. Under these circumstances, direct illumination puts $\sim 10$ times more flux on target and generates $\sim 3$ times the pressure of $\mathbf{X}$-ray illumination. 

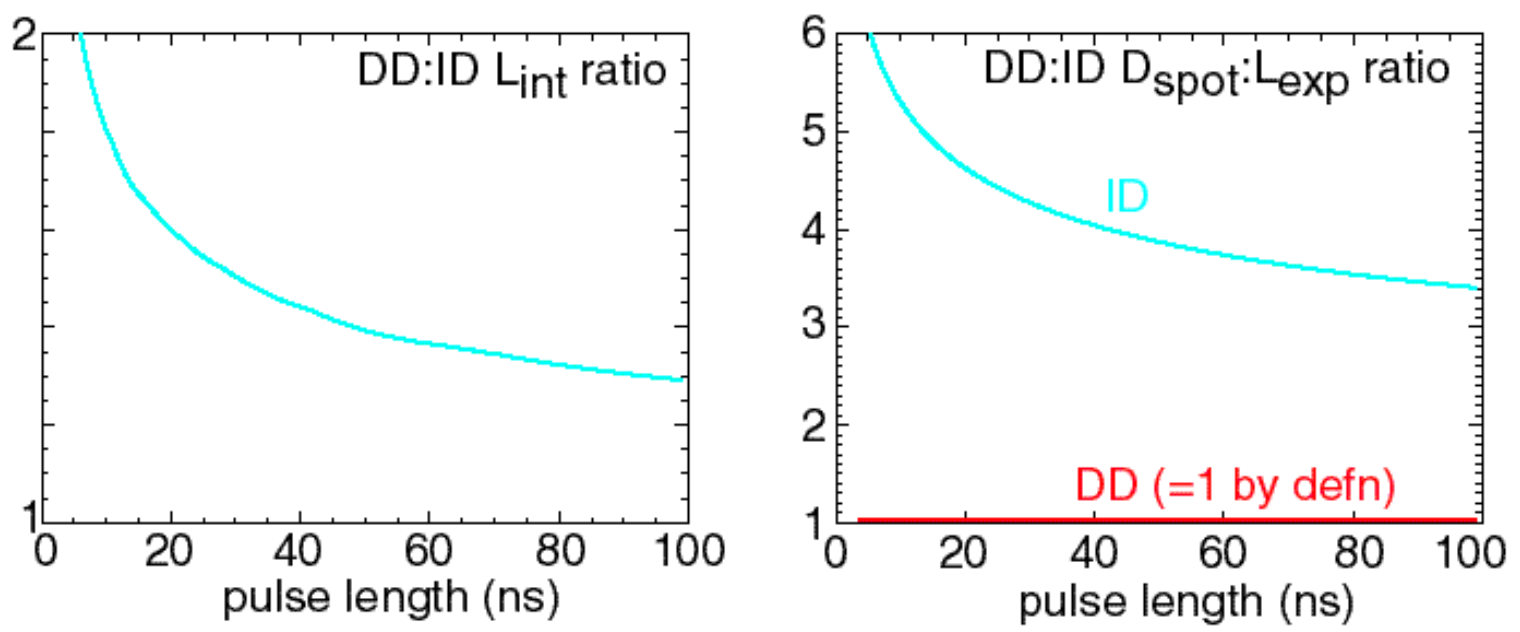

Figure 15 Ratio of direct to indirect drive performance (left) and ratio of spot diameter or hohlraum diameter to length of experiment (right). Most advantage for direct drive is predicted for relatively short pulses. Note that the hohlraum diameter is much greater than the experimental length indicating that $2 \mathrm{D}$ effects should not be problematic for indirect drive.
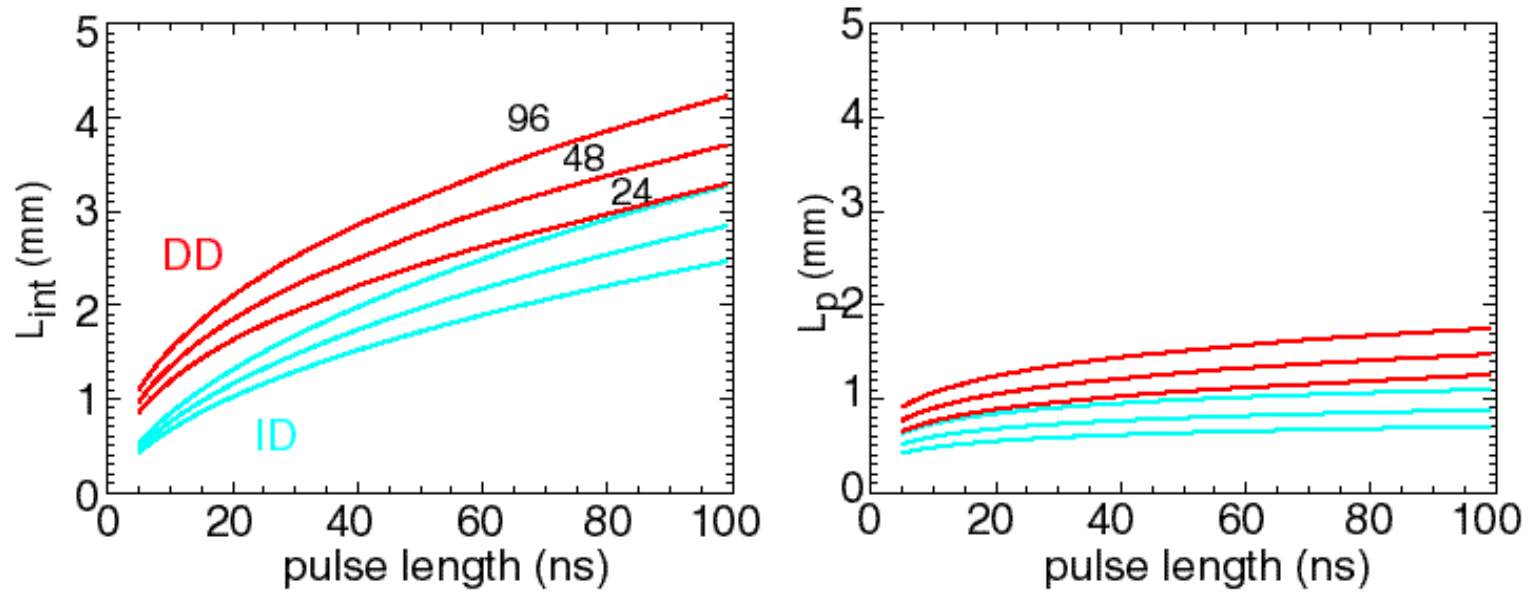

Figure 16a Model predictions of performance and target parameters as a function of available laser energy. 

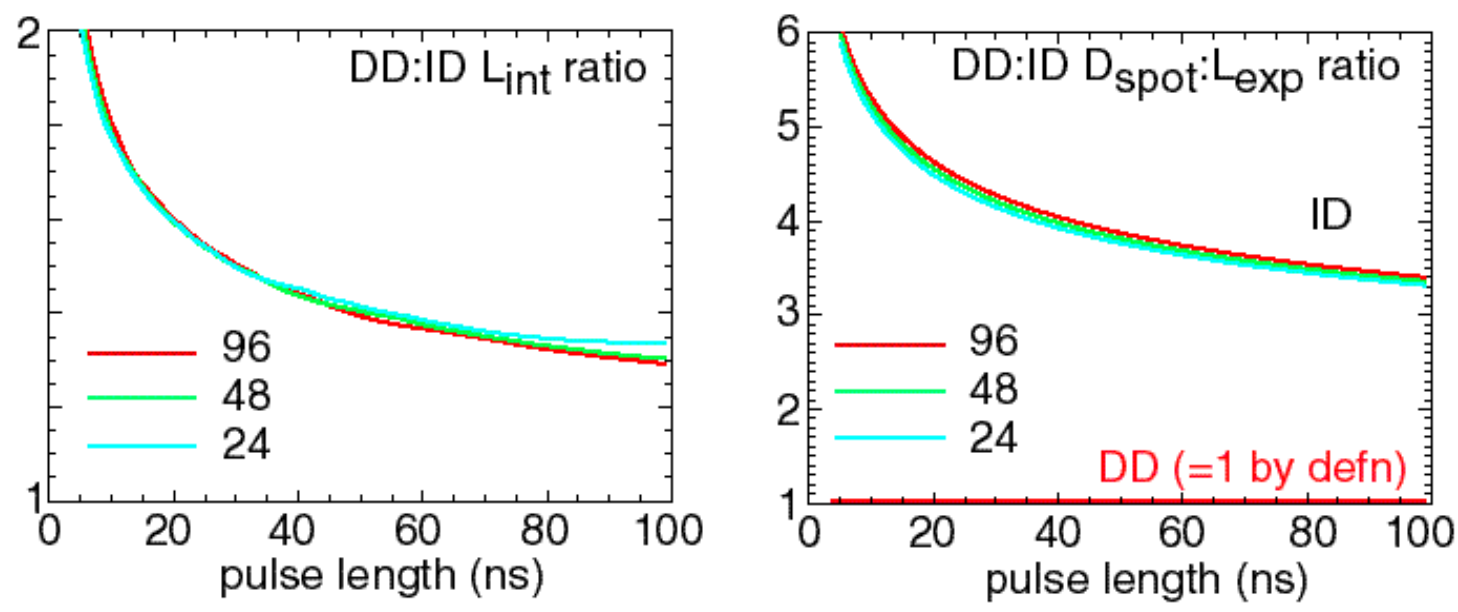

Figure 16b Direct versus indirect drive performance ratios displayed for various drive beam configurations. The relative performance is only very weakly dependent on the available laser energy.

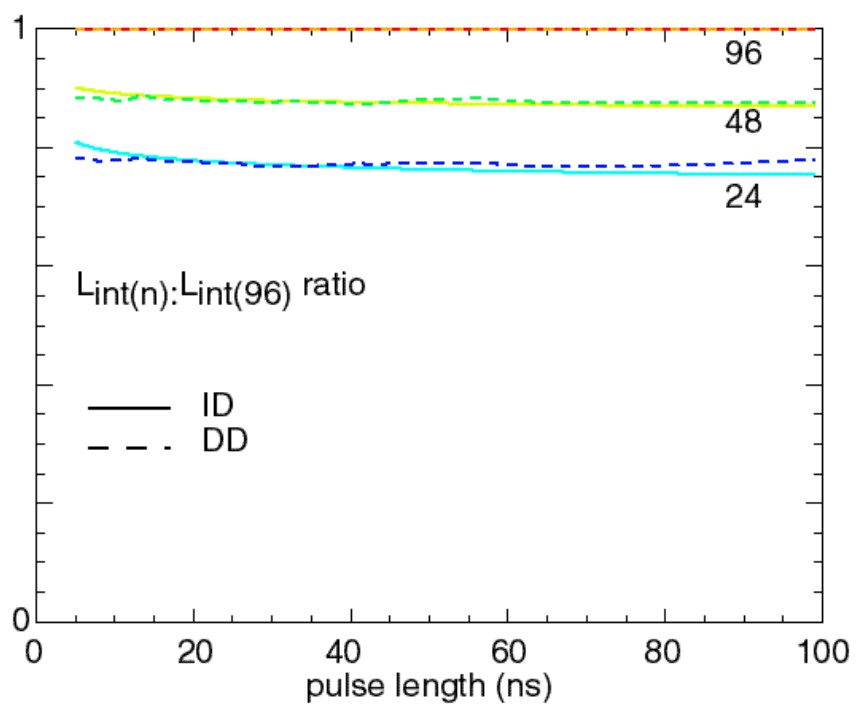

Figure 16c Ratio plot demonstrating the relatively weak dependence of performance on available drive energy. 


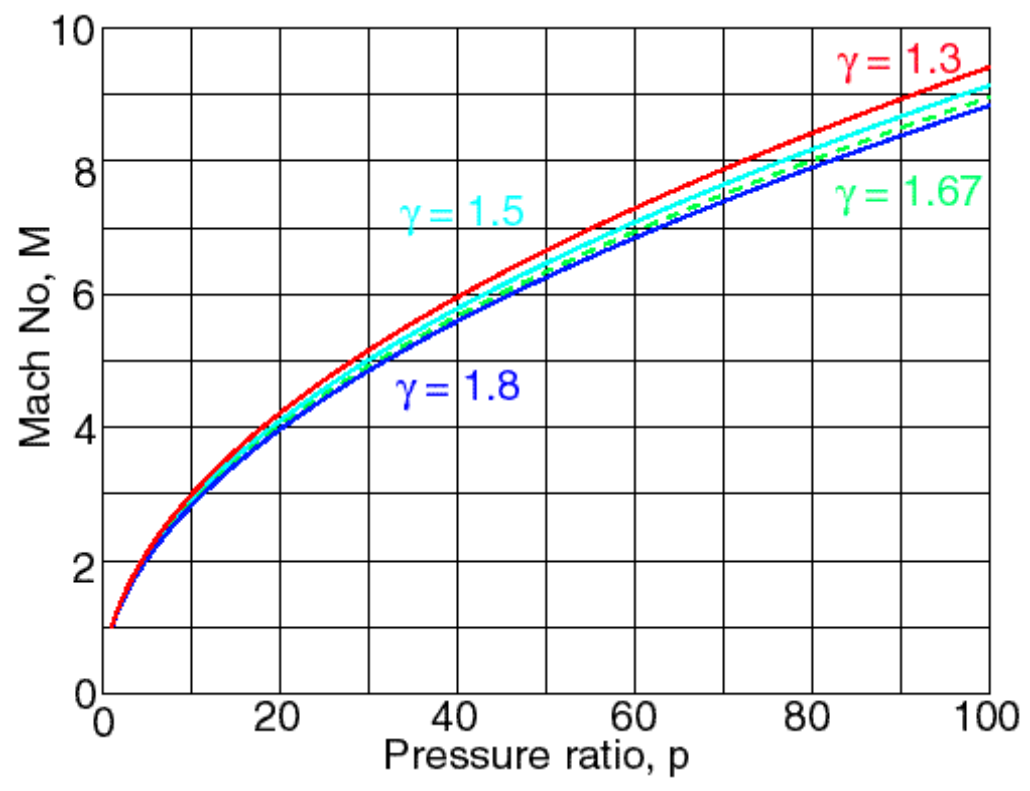

Figure 17 Mach number as a function of post to pre-shock pressure ratio assuming perfect gas EOS for various $\gamma$.

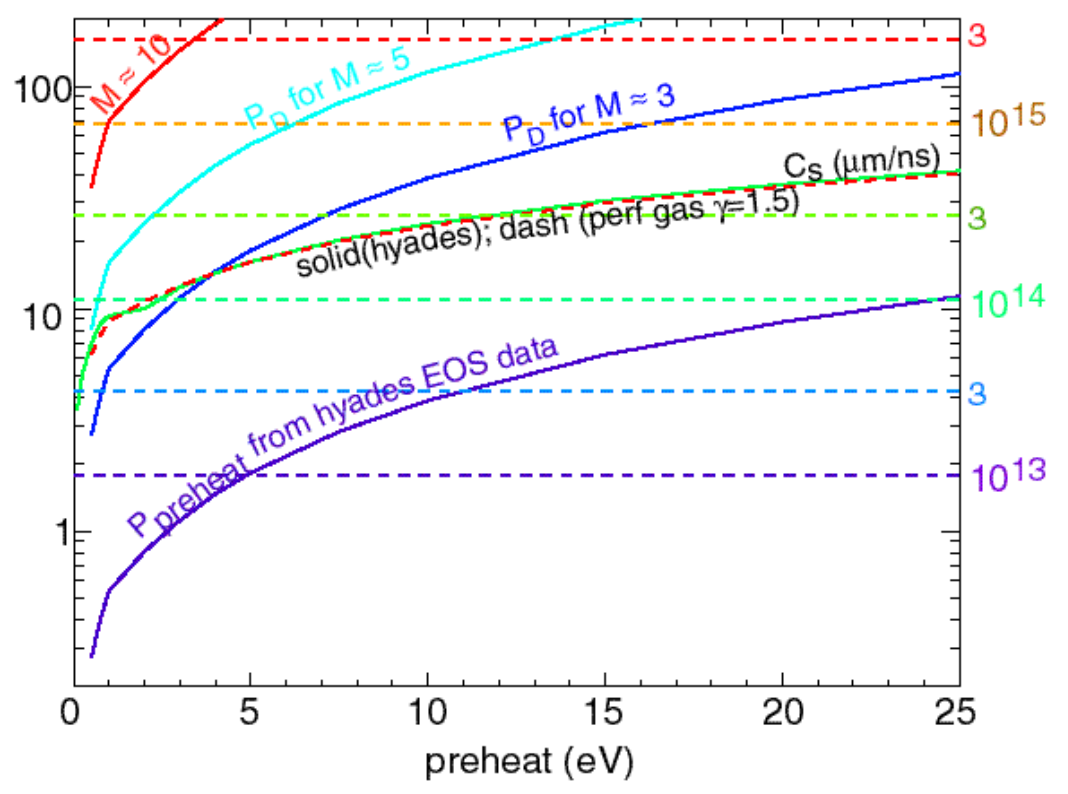

Figure 18 Preheat pressure as a function of preheat temperature from hyades EOS data. Drive pressures required to achieve $M \approx(3,5,10)$ as a function of preheat temperature are shown, based on fig 17 . The hyades sound speed is shown and compared with a perfect gas law. Ablation pressures corresponding to certain intensities are indicated as horizontal lines. 


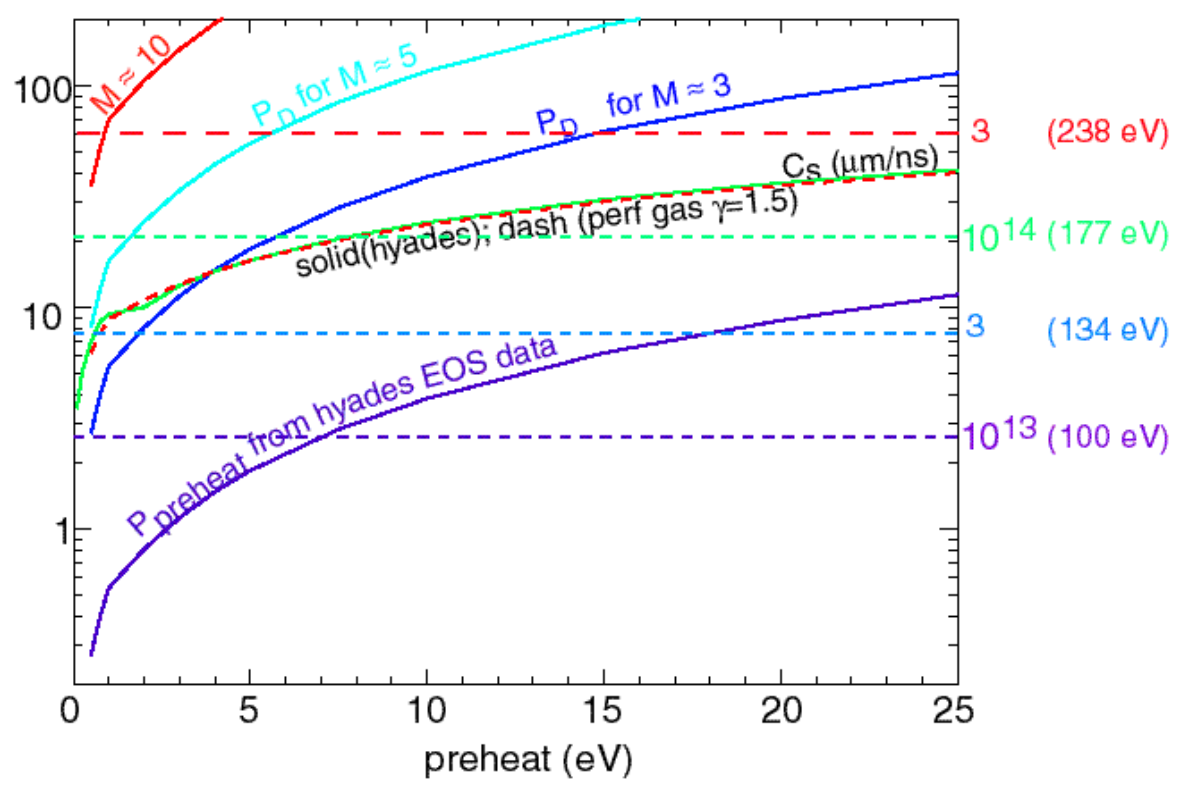

Figure 19 As fig 18 but for indirect drive.

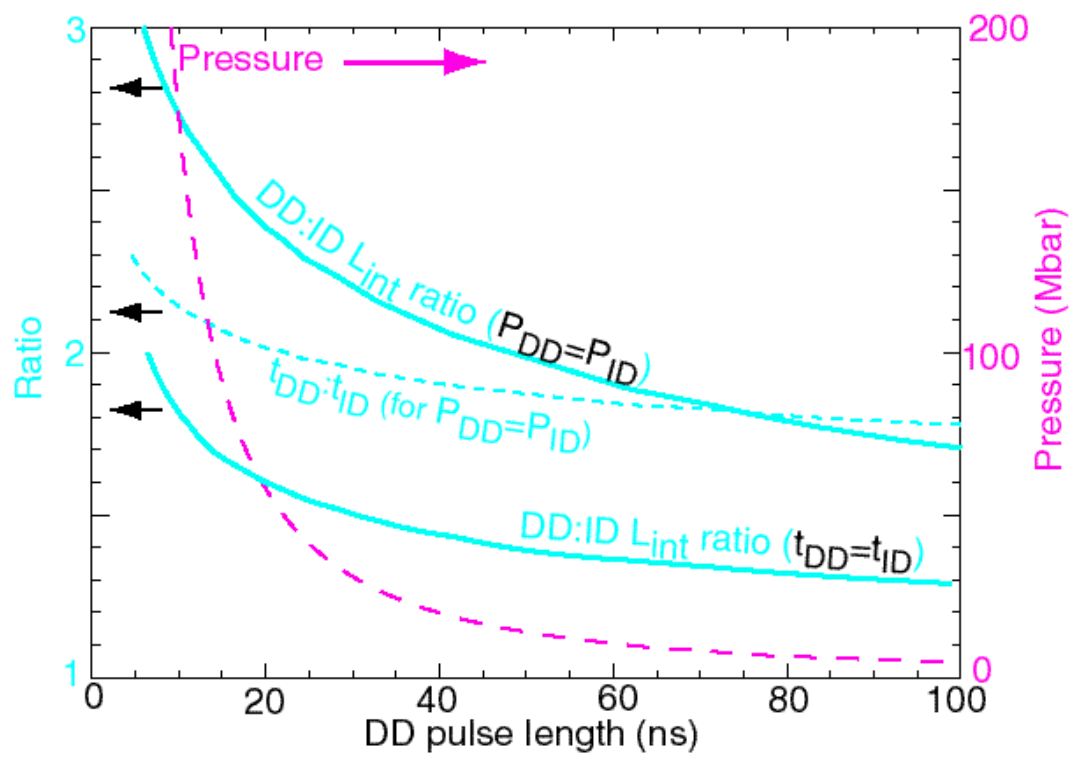

Figure 20 Direct to indirect drive performance ratio at either the same ablation pressure or same pulse length as a function of the direct drive pulse length. For the case of equivalent ablation pressure (shown), the ratio of the direct to indirect drive pulse length is shown, and is approximately 2 . The requirement to operate at the same ablation pressure would exaggerate direct drive's advantage. 